\title{
REPRESENTATION THEORY OF SYMMETRIC GROUPS AND THE STRONG LEFSCHETZ PROPERTY
}

\author{
SEOK-JIN KANG*, YOUNG ROCK KIM**, AND YONG-SU SHIN ${ }^{\dagger}$
}

\begin{abstract}
We investigate the structure and properties of an Artinian monomial complete intersection quotient $A(n, d)=\mathbb{k}\left[x_{1}, \ldots, x_{n}\right] /\left(x_{1}^{d}, \ldots, x_{n}^{d}\right)$. We construct explicit homogeneous bases of $A(n, d)$ that are compatible with the $S_{n}$-module structure for $n=3$, all exponents $d \geq 3$ and all homogeneous degrees $j \geq 0$. Moreover, we derive the multiplicity formulas, both in recursive form and in closed form, for each irreducible component appearing in the $S_{3}$-module decomposition of homogeneous subspaces.
\end{abstract}

\section{INTRODUCTION}

The purpose of this paper is to investigate the structure and properties of an Artinian monomial complete intersection quotient

$$
A(n, d) \cong \mathbb{k}\left[x_{1}, \ldots, x_{n}\right] /\left(x_{1}^{d}, \ldots, x_{n}^{d}\right)=\bigoplus_{j=0}^{m(d)} A(n, d)_{j},
$$

where $\mathbb{k}$ is an algebraically closed field of characteristic 0 and $m(d)=n(d-1)$.

In [16], R. Stanley proved that $A(n, d)$ has the strong Lefschetz property in the narrow sense with $\ell=$ $x_{1}+\cdots+x_{n}$ as a strong Lefschetz element. That is, the linear map $F^{d}=\times \ell^{d}: A_{j} \rightarrow A_{j+d}$ has the maximal rank for all $j \geq 0, d \geq 1$ and its Hilbert polynomial

$$
\operatorname{Hilb}(A(n, d), t):=\sum_{j=0}^{m(d)}\left(\operatorname{dim} A(n, d)_{j}\right) t^{j}
$$

satisfies condition

$$
\operatorname{dim} A(n, d)_{j}=\operatorname{dim} A(n, d)_{m(d)-j} \quad \text { for all } j=0,1, \ldots,\left\lfloor\frac{m(d)}{2}\right\rfloor .
$$

Since it is a major breakthrough in the theory of Lefschetz properties, Stanley's Theorem has been reproved in various contexts. In particular, J. Watanabe took a representation-theoretic approach to this theorem, which attracts our attention.

The symmetric group $S_{n}$ acts on the polynomial algebra $\mathbb{k}\left[x_{1}, \ldots, x_{n}\right]$ by permuting the variables, which induces a natural $S_{n}$-action on $A(n, d)$. Since $\ell=x_{1}+\cdots+x_{n}$ is invariant under the $S_{n}$-action, the linear map $F=\times \ell: A(n, d)_{j} \rightarrow A(n, d)_{j+1}$ commutes with the symmetric group action. Moreover, the linear map

2010 Mathematics Subject Classification. Primary:13A02, Secondary: 20C99.

Key words and phrases. Strong Lefschetz property, Representation theory, Symmetric group, Artinian monomial complete intersection quotients, Hilbert polynomial.

* This research was supported by Hankuk University of Foreign Studies Research Fund.

** This research was supported by the Basic Science Research Program of the NRF (Korea) under grant No. 2015R1D1A1A01059643.

${ }^{\dagger}$ This research was supported by the Basic Science Research Program of the NRF (Korea) under grant No. 2019R1F1A1056934. 
$E: A(n, d)_{j+1} \rightarrow A(n, d)_{j}$, defined by

$$
E\left(x_{1}^{a_{1}} \cdots x_{n}^{a_{n}}\right)=\sum_{k=0}^{n} a_{k}\left(d-a_{k}\right) x_{1}^{a_{1}} \cdots x_{k}^{a_{k}-1} \cdots x_{n}^{a_{n}},
$$

also commutes with the $S_{n}$-action. It is straightforward to verify that the linear maps $F, E, H:=[E, F]$ generate a Lie algebra isomorphic to $\mathfrak{s l}_{2}$. Hence the the algebra $A(n, d)$ has a $\left(\mathbb{k}\left[S_{n}\right] \times U\left(\mathfrak{s l}_{2}\right)\right)$-bimodule structure, where $\mathbb{k}\left[S_{n}\right]$ is the group algebra of $S_{n}$ and $U\left(\mathfrak{s l}_{2}\right)$ is the universal enveloping algebra of $\mathfrak{s l}_{2}$.

One of our main goals of this paper is to give an explicit construction of homogeneous bases of the algebra $A(n, d)$ which is compatible with the $S_{n}$-module structure. As we have seen in the above discussion, this problem is reduced to finding such bases of $\operatorname{Ker}(E) \cap A(n, d)_{j}$ for $j=0,1, \ldots,\left\lfloor\frac{m(d)}{2}\right\rfloor$. We achieve our goal by combining the representation theory of symmentric groups and the standard $\mathfrak{s l}_{2}$-theory. In addition, we determine the multiplicity of each irreducible $S_{n}$-module appearing in the decomposition of homogeneous subspaces $\operatorname{Ker}(E)$. In Section 3, we express these multiplicities in terms of rectangular partitions and in Section 4 , we prove interesting recusrsive relations between the multiplicities. Finally, in Section 5, we derive explicit closed form formulas for the multiplicities. In this paper, we focus on the case when $n=3$, all the exponents $d \geq 3$ and all the homogeneous degrees $j \geq 0$. The more general cases will be dealt with in our forthcoming paper [8].

This paper is organized as follows. In Section 1, we briefly review the standard $\mathfrak{s l}_{2}$-theory and explain the representation-theoretic approach to the Artinian graded $\mathbb{k}$-algebras having the strong Lefschetz property in the narrow sense. In Section 2, we recall some of basic representation theory of symmetric groups and recollect the related combinatorics of Young diagrams and Young tableaux. In Section 3, we explain the strategy to construct explicit homogeneous basis polynomials and determine the multiplicities of irreducible submodules in terms of rectangular partitions. In Section 4 and Section 5, we derive the multiplicity formulas, both in recursive form and in closed form, for each irreducible component appearing in the $S_{3}$-module decomposition of homogeneous subspaces.

\section{Basic $\mathfrak{s l}_{2}$-Theory And Strong Lefschetz Property}

We begin with a brief review of basic $\mathfrak{s l}_{2}$-theory and the properties of graded algebras with strong Lefschetz property in the narrow sense. In this paper, $\mathbb{k}$ denotes an algebraically closed field of characteristic 0.

Recall that $\mathfrak{s l}_{2}$ is the Lie algebra generated by the elements $e, f, h$ with defining relations

$$
[e, f]=h, \quad[h, e]=2 e, \quad[h, f]=-2 f .
$$

For each $m \in \mathbf{Z}_{\geq 0}$, it is well-known that there exists a unique (up to isomorphism) $(m+1)$-dimensional irreducible $\mathfrak{s l}_{2}$-modules $V(m)$ with a basis $\left\{v_{0}, v_{1}, \ldots, v_{m}\right\}$, where the $\mathfrak{s l}_{2}$-action is given by

$$
\begin{aligned}
& h \cdot v_{k}=(m-2 k) v_{k}, \\
& f \cdot v_{k}=v_{k+1}, \\
& e \cdot v_{k}=k\left(m_{k}+1\right) v_{k-1} .
\end{aligned}
$$

Here, we understand $v_{-1}=v_{m+1}=0$.

Let $V$ be a finite-dimensional $\mathfrak{s l}_{2}$-module. We say that a non-zero vector $v \in V$ has weight $\lambda$ if $h \cdot v=\lambda v$ for some $\lambda \in \mathbb{k}$. Moreover, $v$ is called a highest weight vector (resp. lowest weight vector) if $e \cdot v=0$ (resp. $f \cdot v=0)$. Thus, in $V(m), v_{k}$ has weight $m-2 k, v_{0}$ is a highest weight vector with weight $m$ and $v_{m}$ is a lowest weight vector with weight $-m$. 
Over an algebraically closed field of characteristic 0, Weyl's Theorem implies that every finite-dimensional $\mathfrak{s l}_{2}$-module is completely reducible (see, for example, [7]). Let $V$ and $W$ be finite-dimensional $\mathfrak{s l}_{2}$-modules. Then $V \otimes W$ becomes an $\mathfrak{s l}_{2}$-module via

$$
x \cdot(v \otimes w)=x \cdot v \otimes w+v \otimes x \cdot w \quad \text { for } x \in \mathfrak{s l}_{2}, v \in V, w \in W .
$$

For $m \geq n \geq 0$, by Weyl's Theorm, the tensor product $V(m) \otimes V(n)$ is completely reducible and its irreducible decomposition is given by Clebsch-Gordan formula:

$$
V(m) \otimes V(n) \cong V(m+n) \oplus V(m+n-2) \oplus \cdots \oplus V(m-n) .
$$

For example, we have

$$
\begin{aligned}
& V(3) \otimes V(2) \cong V(5) \oplus V(3) \oplus V(1), \\
& V(2) \otimes V(2) \otimes V(2) \cong V(6) \oplus V(4)^{\oplus 2} \oplus V(2)^{\oplus 3} \oplus V(0) .
\end{aligned}
$$

Let $A=\bigoplus_{j=0}^{m} A_{j}$ be a finite-dimensional graded $\mathbb{k}$-algebra. We define its Hilbert polynomial by

$$
\operatorname{Hilb}(A, t):=\sum_{j=0}^{m}\left(\operatorname{dim} A_{j}\right) t^{j} .
$$

We say that $A$ has the strong Lefschetz property in the narrow sense if there exists an element $\ell \in A_{1}$ such that the linear map $F^{d}=\times \ell^{d}: A_{j} \rightarrow A_{j+d}$ has maximal rank for all $j \geq 0, d \geq 1$ and $\operatorname{dim} A_{j}=\operatorname{dim} A_{m-j}$ for $0 \leq j \leq\left\lfloor\frac{m}{2}\right\rfloor$. In this case, $\ell$ is called a strong Lefschetz element.

Since $A$ is finite-dimensional, the linear map $F=\times \ell: A \rightarrow A$ is nilpotent. Consider the Jordan canonical form of $F$ with Jordan blocks of size $m_{1}, \ldots, m_{r}$. We rearrange the Jordan blocks so that we have $m_{1} \geq m_{2} \geq$ $\cdots \geq m_{r}>0$. For each $j=1, \ldots, r$, let $B_{j}=\left\{u_{j}, F\left(u_{j}\right), \ldots, F^{m_{j}-1}\left(u_{j}\right)\right\}$ be a Jordan canonical basis of the corresponding Jordan block. By our construction, $B:=\sqcup_{j=1}^{r} B_{j}$ is a Jordan canonical basis of $A$ with respect to $F$.

For each $j=1, \ldots, r$, let $A^{(j)}$ be the subspace of $A$ spanned by $B_{j}$ and define the linear maps $H: A^{(j)} \rightarrow$ $A^{(j)}, E: A^{(j)} \rightarrow A^{(j)}$ by

$$
H\left(F^{k}\left(u_{j}\right)\right)=\left(m_{j}-2 k\right) F^{k}\left(u_{j}\right), \quad E\left(F^{k}\left(u_{j}\right)\right)=k\left(m_{j}-k\right) F^{k-1}\left(u_{j}\right) \quad\left(0 \leq k \leq m_{j}-1\right) .
$$

Then it is straightforward to verify that $E, F, H$ satisfy the defining relation (1.1) and for each $j=1, \ldots, r$, the vectors $F^{k}\left(u_{j}\right)$ satisfy the relations in (1.2). It follows that each subspace $A^{(j)}$ is isomorphic to the irreducible $\mathfrak{s l}_{2}$-module $V\left(m_{j}-1\right)$.

Hence $A$ becomes a completely reducible $\mathfrak{s l}_{2}$-module and its irreducible decomposition is determined by the Jordan canonical form of $F$. More precisely, we have

$$
A \cong \bigoplus_{j=0}^{\left\lfloor\frac{m}{2}\right\rfloor} V(m-2 j)^{\oplus a_{j}}
$$

where $a_{0}=1, a_{j}=\operatorname{dim} A_{j}-\operatorname{dim} A_{j-1}$ for $0 \leq j \leq\left\lfloor\frac{m}{2}\right\rfloor$.

Example 1.1. Let $A=\mathbb{k}\left[x_{1}, x_{2}, x_{3}\right] /\left(x_{1}^{3}, x_{2}^{3}, x_{3}^{3}\right)$. Then its Hilbert polynomial is

$$
\operatorname{Hilb}(A, t)=1+3 t+6 t^{2}+7 t^{3}+6 t^{4}+3 t^{5}+t^{6} .
$$

Hence the $\mathfrak{s l}_{2}$-decomposition of $A$ is given by

$$
A \cong V(6) \oplus V(4)^{\oplus 2} \oplus V(2)^{\oplus 3} \oplus V(0)
$$

Note that $A \cong V(2)^{\otimes 3}$ as an $\mathfrak{s l}_{2}$-module. 
The following proposition is one of the main results in [4]. The key ingredient of its proof is the ClebschGordan formula.

Proposition $1.2([4])$. Let $\left(A_{1}, \ell_{1}\right)$ and $\left(A_{2}, \ell_{2}\right)$ be finite dimensional graded algebras having the strong Lefschetz property in the narrow sense with strong Lefschetz elements $\ell_{1}$ and $\ell_{2}$, respectively. Then $A_{1} \otimes A_{2}$ also has the strong Lefschetz property in the narrow sense with a strong Lefschetz element $\ell=\ell_{1} \otimes 1+1 \otimes \ell_{2}$.

As an immediate corollary, we obtain a representation-theoretic proof of Stanley's Theorem.

Corollary $1.3([4,17])$. An Artinian monomial complete intersection quotient

$$
A=\mathbb{k}\left[x_{1}, \ldots, x_{n}\right] /\left(x_{1}^{d_{1}}, \ldots, x_{n}^{d_{n}}\right)
$$

has the strong Lefschetz property in the narrow sense with a strong Lefschetz element $\ell=x_{1}+\cdots+x_{n}$.

\section{REPRESENTATION THEORY OF SYMMETRIC GROUPS}

We now recollect some pieces of the representation theory of $S_{n}$. A weakly decreasing sequence of positive integers $\lambda=\left(\lambda_{1} \geq \lambda_{2} \geq \cdots \geq \lambda_{r}>0\right)$ is called a partition of $n$ if $\lambda_{1}+\lambda_{2}+\cdots+\lambda_{r}=n$. The positive integer $r$ is called the length of $\lambda$, usually written as $\ell(\lambda)$. We denote by $\mathscr{P}(n)$ the set of all partitions of $n$ and $p(n)$ the number of elements in $\mathscr{P}(n)$. A partition $\lambda=\left(\lambda_{1} \geq \lambda_{2} \geq \cdots \geq \lambda_{r}>0\right)$ is called a rectangular partition of siz $k \times l$ if $\ell(\lambda) \leq k$ and all the entries in $\lambda$ are $\leq l$. We denote by $\mathscr{P}_{k, l}(n)$ the set of all rectangular partitions of $n$ of size $k \times l$ and $p_{k, l}(n)$ the number of elements in $\mathscr{P}(n)$.

We identify a partition $\lambda=\left(\lambda_{1} \geq \lambda_{2} \geq \cdots \geq \lambda_{r}>0\right)$ with the Young diagram $Y^{\lambda}$ which consists of $\lambda_{1^{-}}$ many boxes in the first row, $\lambda_{2}$-many boxes in the second row, etc. For instance, the partition $\lambda=(5,3,2,2)$ of 12 is identified with the following Young diagram.

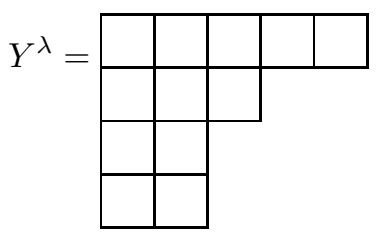

Definition 2.1. Let $\lambda$ be a partition of $n$. A standard tableau of shape $\lambda$ is a filling of the boxes of $Y^{\lambda}$ with entries taken from $1,2, \ldots, n$ such that

(i) all the entries in each row are strictly increasing from left to right,

(ii) all the entries in each column are strictly increasing from top to bottom.

An example of standard tableau of shape $\lambda=(5,3,2,2)$ is given below.

$$
T=\begin{array}{|c|c|c|c|c|}
\hline 1 & 2 & 4 & 6 & 9 \\
\hline 3 & 8 & 11 & \multicolumn{3}{|l}{} \\
\cline { 1 - 2 } 5 & 10 & \multicolumn{3}{|l}{} \\
\cline { 1 - 2 } 7 & 12 & \multicolumn{3}{|l}{} \\
\cline { 1 - 1 }
\end{array}
$$

To each partition $\lambda$ of $n$, there exists a unique (up to isomorphism) irreducible $S_{n}$-module $S^{\lambda}$ which is called the Specht module associated with $\lambda$. It is known that every finite dimensional irreducible $S_{n}$-module has the form $S^{\lambda}$ for some partition $\lambda$ of $n$. Furthermore, the dimension of $S^{\lambda}$ is given by the number of standard tableaux of shape $\lambda$. (See, for example, $[1,2,14]$ ). 
There are three special types of irreducible $S_{n}$-modules: trivial representation, sign representation and standard representation.

(a) The trivial representation is the 1-dimensional $S_{n}$-modules on which every permutaton acts as the identity. One can see that the trivial representation corresponds to the partition $\lambda=(n)$.

(b) The sign representation is the 1-dimensional $S_{n}$-module on which every permutation acts as the multiplication by its sign. The sign representation corresponds to the partition $\lambda=(1,1, \ldots, 1)$.

(c) The standard module is the Specht module corresponding to the partition $\lambda=(n-1,1)$. It can be identified with the $(n-1)$-dimensional hyperplane $H=\left\{\left(x_{1}, \ldots, x_{n}\right) \in \mathbb{k}^{n} \mid x_{1}+\cdots+x_{n}=0\right\}$.

When $n=3$, they are all ireducible representations of the symmetric group.

Example 2.2. In the following figure, we exhibit all the standard tableaux corresponding to the partitions $(3),(1,1,1)$ and $(2,1)$.

$$
\begin{aligned}
& \lambda=(3), \quad \begin{array}{|l|l|l|}
\hline 1 & 2 & 3 \\
\hline
\end{array} \\
& \lambda=(1,1,1), \begin{array}{|l|}
\hline 1 \\
\hline 2 \\
\hline 3 \\
\hline
\end{array} \\
& \lambda=(2,1), \quad \begin{array}{|l|l|l|l|}
\hline 1 & 2 \\
\hline 3 &
\end{array}, \begin{array}{|l|l|}
\hline 1 & 3 \\
\hline 2 & \multicolumn{1}{|l}{.}
\end{array} \text {. }
\end{aligned}
$$

Hence $\operatorname{dim} S^{(3)}=\operatorname{dim} S^{(1,1,1)}=1$ and $\operatorname{dim} S^{(2,1)}=2$.

\section{ARTinian monomial COMPLETE INTERSECTION QUOTIENTS}

Let $A:=A(n, d)=\mathbb{k}\left[x_{1}, \ldots, x_{n}\right] /\left(x_{1}^{d}, \ldots, x_{n}^{d}\right)=A_{0} \oplus A_{1} \oplus \cdots \oplus A_{m}$ be an Artinian monomial complete intersection quotient with the same exponent $d$, where $m=n(d-1)$. Recall that the symmetric group $S_{n}$ acts on $\mathbb{k}\left[x_{1}, \ldots, x_{n}\right]$ by permuting the variables. Since the generators have the same exponents, we have a natural $S_{n}$-action on $A$. We have seen that $A$ has the strong Lefschetz property in the narrow sense with a strong Lefschetz element $\ell=x_{1}+\cdots+x_{n}$. Define a linear map $E: A_{j+1} \rightarrow A_{j}$ by

$$
E\left(x_{1}^{a_{1}} \cdots x_{n}^{a_{n}}\right)=\sum_{k=1}^{n} a_{k}\left(d-a_{k}\right) x_{1}^{a_{1}} \cdots x_{k}^{a_{k}-1} \cdots x_{n}^{a_{n}} .
$$

Then the linear maps $E, F=\times \ell, H:=[E, F]$ generate a Lie algebra which is isomorphic to $\mathfrak{s l}_{2}$.

Clearly, each homogeneous subspace $A_{j}$ is invariant under the $S_{n}$-action on $A$. Since $\ell=x_{1}+\cdots+x_{n}$ is $S_{n}$-invariant, we have $F \circ \sigma=\sigma \circ F$ for all $\sigma \in S_{n}$. Furthermore, it can be shown that $E \circ \sigma=\sigma \circ E$ for all $\sigma \in S_{n}$ (cf. [4]). That is, $E$ and $F$ are $S_{n}$-module homomorphisms.

One of the main goals of this paper is to construct explicit homogeneous bases of irreducible $S_{n}$-submodules that appear as a direct summand in the $S_{n}$-module decomposition of $A$. Since $A$ is a completely reducible $\mathfrak{s l}_{2}$-module, by Schur's Lemma, it suffices to construct such bases for $\operatorname{Ker}(E) \cap A_{j}\left(j=0,1, \ldots,\left\lfloor\frac{m}{2}\right\rfloor\right)$. For general $n, d$ and $j$, it is not yet known how to construct such an explicit basis directly. (In [10], Maeno gave an inductive algorithm.) We expect the general case can be solved using the Young tabloid construction of Specht modules [8]. 
In this paper, we focus on the case when $n=3$ and deal with all the exponents $d \geq 3$ and all the homogeneous degrees $j \geq 0$. Recall that all the irreducible $S_{3}$-modules are classified as one of the following: trivial representation, sign representaion and standard representation. We give an explicit construction of homogeneous bases for these irreducible $S_{3}$-submodules of $\operatorname{Ker}(E) \cap A_{j}\left(j=0,1, \ldots,\left\lfloor\frac{m}{2}\right\rfloor\right)$. Moreover, we determine their multiplicities in the irreducible decomposition of $\operatorname{Ker}(E)$ in each homogeneous subspace of $A$.

For simplicity, we will write $A(d)$ for $A(n, d)$ and denote

$$
\operatorname{mult}(d, j)=\operatorname{dim}\left(\operatorname{Ker}(E) \cap A(d)_{j}\right)=\operatorname{dim} A(d)_{j}-\operatorname{dim} A(d)_{j-1},
$$

the multiplicity of the irreducible highest weight $\mathfrak{s l}_{2}$-module $V(3(d-1)-2 j)$ in $A(d)_{j}$. Then using the Hilbert polynomial of $A(d)$, we obatin

$$
\operatorname{mult}(d, j)= \begin{cases}j+1 & \text { for } 0 \leq j \leq d-1 \\ 3 d-2-2 j & \text { for } \quad d \leq j \leq\left\lfloor\frac{3(d-1)}{2}\right\rfloor\end{cases}
$$

Now we state and prove our main results.

\section{(1) Trivial representation:}

Given $d$ and $j$, since $S_{3}$ acts trivially, any basis polynomial of the trivial representation in $A(d)_{j}$ is a homogeneous symmetric polynomial of degree $j$, say, $P$. Hence it can be expressed as

$$
P=\sum_{\lambda=(a, b, c)} \alpha_{a, b, c}\left[x_{1}^{a} x_{2}^{b} x_{3}^{c}\right]
$$

where $\lambda=(a, b, c)$ is a partition of $j$ of size $3 \times(d-1)$ and $\left[x_{1}^{a} x_{2}^{b} x_{3}^{c}\right]$ denotes the $S_{3}$-orbit of the monomial $x_{1}^{a} x_{2}^{b} x_{3}^{c}$ (under the permutation action of variables). For instance, when $d \geq 4$, we have

$$
\begin{aligned}
& {\left[x_{1}^{3}\right]=x_{1}^{3}+x_{2}^{3}+x_{3}^{3},} \\
& {\left[x_{1}^{2} x_{2}\right]=x_{1}^{2} x_{2}+x_{1}^{2} x_{3}+x_{2}^{2} x_{3}+x_{1} x_{2}^{2}+x_{1} x_{3}^{2}+x_{2} x_{3}^{2},} \\
& {\left[x_{1} x_{2} x_{3}\right]=x_{1} x_{2} x_{3} .}
\end{aligned}
$$

Then the condition $E(P)=0$ would yield a system of linear equatons with variables $\alpha_{a, b, c}$. The solutions of this system give the desired explicit basis polynomials and the nullity of the coefficient matrix is the multiplicity of the trivial representation in $\operatorname{Ker}(E) \cap A(d)_{j}$. Note that the number of variables is the same as $p_{3, d-1}(j)$ and the number of equations is $p_{3, d-1}(j-1)$. Indeed, let $\mathscr{P}_{3, d-1}(j)=\{\lambda \mid \lambda=(a, b, c), d>a \geq b \geq c \geq 0\}$ with $a+b+c=j$. So the number of variables in $E(P)=0$ is $p_{3, d-1}(j)=\left|\mathscr{P}_{3, d-1}(j)\right|$. Moreover, notice that $\operatorname{deg} E(P)=j-1$. Let $\mathscr{P}_{3, d-1}(j)=\left\{\lambda_{1}, \ldots, \lambda_{s}\right\}$ and let $\mu_{1}=(1,0,0), \mu_{2}=(0,1,0), \mu_{3}=(0,0,1)$. Then the collection of all partitions of the form $\lambda_{i}-\mu_{j}$ is $\mathscr{P}_{3, d-1}(j-1)$ (here $\lambda_{i}-\mu_{j}$ is not necessarily a partition). In other words, the number of equations from $E(P)=0$ is $p_{3, d-1}(j-1)=\left|\mathscr{P}_{3, d-1}(j-1)\right|$.

Example 3.1. Let $n=3, d=4$, and $j=3$. Then $\mathscr{P}_{3,3}(3)=\{(3,0,0),(2,1,0),(1,1,1)\}$ and let

$$
\begin{aligned}
P & =\alpha_{3,0,0}\left[x_{1}^{3}\right]+\alpha_{2,1,0}\left[x_{1}^{2} x_{2}\right]+\alpha_{1,1,1}\left[x_{1} x_{2} x_{3}\right] \\
& =\alpha_{3,0,0}\left(x_{1}^{3}+x_{2}^{3}+x_{3}^{3}\right)+\alpha_{2,1,0}\left(x_{1}^{2} x_{2}+x_{1}^{2} x_{3}+x_{2}^{2} x_{3}+x_{1} x_{2}^{2}+x_{1} x_{3}^{2}+x_{2} x_{3}^{2}\right)+\alpha_{1,1,1}\left(x_{1} x_{2} x_{3}\right) .
\end{aligned}
$$

So $p_{3,3}(3)=|\{(3,0,0),(2,1,0),(1,1,1)\}|=3$ unknowns. Furthermore,

$$
\begin{aligned}
E(P) & =3 \alpha_{3,0,0}\left[x_{1}^{2}\right]+4 \alpha_{2,1,0}\left[x_{1} x_{2}\right]+3 \alpha_{2,1,0}\left[x_{1}^{2}\right]+3 \alpha_{2,1,0}\left[x_{1} x_{2}\right] \\
& =\left(3 \alpha_{3,0,0}+3 \alpha_{2,1,0}\right)\left[x_{1}^{2}\right]+\left(4 \alpha_{2,1,0}+3 \alpha_{1,1,1}\right)\left[x_{1} x_{2}\right] \\
& =0
\end{aligned}
$$

yields two equations, which is $p_{3,3}(2)=|\{(2,0,0),(1,1,0)\}|$. 
The following lemma is easy to prove, but we introduce the proof for completeness.

Lemma 3.2. With notation as above, let $\mu=(a, b, c) \in \mathscr{P}_{3, d-1}(j-1)$. Then at least one of $\mu+(1,0,0), \mu+$ $(0,1,0), \mu+(0,0,1)$ is a partition in $\mathscr{P}_{3, d-1}(j)$.

Proof. First note that since $j \leq\left\lfloor\frac{3(d-1)}{2}\right\rfloor<3(d-1)$, we get that $c<d-1$. If $c<b$, then $\mu+(0,0,1)=(a, b, c+1)$ is a partition in $\mathscr{P}_{3, d-1}(j)$. If $b=c<a$, then $\mu+(0,1,0)=(a, b+1, c)$ is a partition in $\mathscr{P}_{3, d-1}(j)$. If $a=b=c<d-1$, then $\mu+(1,0,0)=(a+1, b, c)$ is a partition in $\mathscr{P}_{3, d-1}(j)$ as well.

For $\lambda=(a, b, c)$ with $d>a \geq b \geq c \geq 0$, we denote $x^{\lambda}=x_{1}^{a} x_{2}^{b} x_{3}^{c}$ and write

$$
E\left(\left[x^{\lambda}\right]\right)=E\left(\left[x_{1}^{a} x_{2}^{b} x_{3}^{c}\right]\right):=C_{\mu_{1}, \lambda}\left[x_{1}^{a-1} x_{2}^{b} x_{3}^{c}\right]+C_{\mu_{2}, \lambda}\left[x_{1}^{a} x_{2}^{b-1} x_{3}^{c}\right]+C_{\mu_{3}, \lambda}\left[x_{1}^{a} x_{2}^{b} x_{3}^{c-1}\right],
$$

where $\mu_{1}=(a-1, b, c), \mu_{2}=(a, b-1, c)$, and $\mu_{3}=(a, b, c-1)$. Note that if $\mu_{i}$ is not a partition, then we have $C_{\mu_{i}, \lambda}=0$.

In general, let $\mathscr{P}_{3, d-1}(j)=\left\{\lambda_{1}, \ldots, \lambda_{t}\right\}$ be the collection of partitions of $j$ and let $\mathscr{P}_{3, d-1}(j-1)=$ $\left\{\mu_{1}, \ldots, \mu_{s}\right\}$ be the collection of partitions of $j-1$ listed by the lexicographic ordering. Thus $t=p_{3, d-1}(j)$ and $s=p_{3, d-1}(j-1)$. Let $P=\alpha_{1}\left[x^{\lambda_{1}}\right]+\cdots+\alpha_{t}\left[x^{\lambda_{t}}\right]$ be a homogeneous symmetric polynomial of degree $j$. Then the condition $E(P)=0$ yields a system of linear equations in the variable $\alpha_{1}, \ldots, \alpha_{t}$ with coefficient matrix $C=\left(C_{p q}\right)$ for $(1 \leq p \leq s, 1 \leq q \leq t)$, where $C_{p q}:=C_{\mu_{p}, \lambda_{q}}$ such that

$$
E\left(\alpha_{q}\left[x^{\lambda_{q}}\right]\right)=\sum_{p=1}^{s} \alpha_{q} C_{\mu_{p}, \lambda_{q}}\left[x^{\mu_{p}}\right]:=\sum_{p=1}^{s} \alpha_{q} C_{p q}\left[x^{\mu_{p}}\right] .
$$

Observe that the coefficients $C_{p q}=0$ unless $\lambda_{q}=\mu_{p}+(1,0,0), \lambda_{q}=\mu_{p}+(0,1,0)$, or $\lambda_{q}=\mu_{p}+(0,0,1)$ and they are partitions. In other words, $C_{p q}=0$ unless the term $\left[x^{\mu_{p}}\right]$ arises as an image of $\left[x^{\mu_{p}+(1,0,0)}\right],\left[x^{\mu_{p}+(0,1,0)}\right]$, or $\left[x^{\mu_{p}+(0,0,1)}\right]$ under $E$. Also note that at least one of them is a partition.

From this observation, we have

$$
\begin{aligned}
& C_{\mu_{1}, \lambda_{1}} \alpha_{1}\left[x^{\mu_{1}}\right]+\cdots+C_{\mu_{s}, \lambda_{1}} \alpha_{1}\left[x^{\mu_{s}}\right]+ \\
& C_{\mu_{1}, \lambda_{2}} \alpha_{2}\left[x^{\mu_{1}}\right]+\cdots+C_{\mu_{s}, \lambda_{2}} \alpha_{2}\left[x^{\mu_{s}}\right]+ \\
& \vdots \\
& C_{\mu_{1}, \lambda_{t}} \alpha_{t}\left[x^{\mu_{1}}\right]+\cdots+C_{\mu_{s}, \lambda_{t}} \alpha_{t}\left[x^{\mu_{s}}\right] \\
= & \left(C_{\mu_{1}, \lambda_{1}} \alpha_{1}+C_{\mu_{1}, \lambda_{2}} \alpha_{2}+\cdots+C_{\mu_{1}, \lambda_{t}} \alpha_{t}\right)\left[x^{\mu_{1}}\right]+ \\
& \left(C_{\mu_{2}, \lambda_{1}} \alpha_{1}+C_{\mu_{2}, \lambda_{2}} \alpha_{2}+\cdots+C_{\mu_{2}, \lambda_{t}} \alpha_{t}\right)\left[x^{\mu_{2}}\right]+ \\
& \vdots \\
& \left(C_{\mu_{s-1}, \lambda_{1}} \alpha_{1}+C_{\mu_{s-1}, \lambda_{2}} \alpha_{2}+\cdots+C_{\mu_{s-1}, \lambda_{t}} \alpha_{t}\right)\left[x^{\mu_{s-1}}\right]+ \\
& \left(C_{\mu_{s}, \lambda_{1}} \alpha_{1}+C_{\mu_{s}, \lambda_{2}} \alpha_{2}+\cdots+C_{\mu_{s}, \lambda_{t}} \alpha_{t}\right)\left[x^{\mu_{s}}\right] \\
= & 0 .
\end{aligned}
$$

Hence we obtain the following linear system

$$
\begin{gathered}
C_{11} \alpha_{1}+\cdots+C_{1 t} \alpha_{t}=0, \\
C_{21} \alpha_{1}+\cdots+C_{2 t} \alpha_{t}=0, \\
\vdots \\
C_{s 1} \alpha_{1}+\cdots+C_{s t} \alpha_{t}=0 .
\end{gathered}
$$

Let $C(a)$ be the submatrix of $C$ such that i) the non-zero entries are determined by the partitions $\mu \in$ $\mathscr{P}_{3, d-1}(j-1), \lambda \in \mathscr{P}_{3, d-1}(j)$, where the first component of $\mu$ is $a$ and the first component of $\lambda$ is $a$ or $a+1$, ii) the other entries are 0 . 
We now consider the following example.

Example 3.3. (a) Let $d=3, j=3$. Then $\mathscr{P}_{3, d-1}(3)=\left\{\lambda_{1}=(2,1,0), \lambda_{2}=(1,1,1)\right\}$ and $\mathscr{P}_{3, d-1}(2)=\left\{\mu_{1}=\right.$ $\left.(2,0,0), \mu_{2}=(1,1,0)\right\}$. The basis element of the trivial representation $P$ can be written as

$$
P=\alpha_{1}\left[x^{\lambda_{1}}\right]+\alpha_{2}\left[x^{\lambda_{2}}\right]=\alpha_{1}\left[x_{1}^{2} x_{2}\right]+\alpha_{2}\left[x_{1} x_{2} x_{3}\right] .
$$

Hence we have

$$
\begin{aligned}
E\left(\left[x^{\lambda_{1}}\right]\right) & =E\left(x_{1}^{2} x_{2}+x_{1}^{2} x_{3}+x_{1} x_{2}^{2}+x_{1} x_{3}^{2}+x_{2}^{2} x_{3}+x_{2} x_{3}^{2}\right) \\
& =2 x_{1} x_{2}+2 x_{1}^{2}+2 x_{1} x_{3}+2 x_{1}^{2}+2 x_{2}^{2}+2 x_{1} x_{2}+2 x_{3}^{2}+2 x_{1} x_{3}+2 x_{2} x_{3}+2 x_{2}^{2}+2 x_{3}^{2}+2 x_{2} x_{3} \\
& =4\left[x^{\mu_{1}}\right]+4\left[x^{\mu_{2}}\right], \quad \text { and } \\
E\left(\left[x^{\lambda_{2}}\right]\right) & =E\left(x_{1} x_{2} x_{3}\right) \\
& =2 x_{1} x_{2}+2 x_{1} x_{3}+2 x_{2} x_{3} \\
& =2\left[x^{\mu_{2}}\right] .
\end{aligned}
$$

Therefore we get

$$
\begin{aligned}
& C_{\mu_{1}, \lambda_{1}}=C_{11}=4, \\
& C_{\mu_{1}, \lambda_{2}}=C_{12}=0, \\
& C_{\mu_{2}, \lambda_{1}}=C_{21}=4, \\
& C_{\mu_{2}, \lambda_{2}}=C_{22}=2 .
\end{aligned}
$$

This means that

$$
C=\left(\begin{array}{ll}
C_{11} & C_{12} \\
C_{21} & C_{22}
\end{array}\right)=\left(\begin{array}{ll}
4 & 0 \\
4 & 2
\end{array}\right)
$$

In other words, the linear system

$$
C\left(\begin{array}{l}
a_{1} \\
a_{2}
\end{array}\right)=0
$$

has only the trivial solution. Hence there is no trivial representation in $\operatorname{Ker}(E) \cap A(3)_{3}$. Note that $p_{3,3}(3)-$ $p_{3,3}(2)=2-2=0$.

Now we have

$$
C=\left(\begin{array}{ll}
C_{11} & C_{12} \\
C_{21} & C_{22}
\end{array}\right)=\left(\begin{array}{ll}
C_{\mu_{1}, \lambda_{1}} & C_{\mu_{1}, \lambda_{2}} \\
C_{\mu_{2}, \lambda_{1}} & C_{\mu_{2}, \lambda_{2}}
\end{array}\right)=\left(\begin{array}{cc}
C_{(2,0,0),(2,1,0)} & 0 \\
C_{(1,1,0),(2,1,0)} & C_{(1,1,0),(1,1,1)}
\end{array}\right)=\left(\begin{array}{l}
C(2) \\
C(1)
\end{array}\right)
$$

and then the rank of $C$ is equal to 2 which is a sum of the number of rows of $C(a), a=2,1$.

(b) Let $d=7, j=6$. Then we have

$$
\mathscr{P}_{3, d-1}(6)=\left\{\lambda_{1}=(6,0,0), \lambda_{2}=(5,1,0), \lambda_{3}=(4,2,0), \lambda_{4}=(4,1,1), \lambda_{5}=(3,3,0), \lambda_{6}=(3,2,1), \lambda_{7}=(2,2,2)\right\}
$$

and

$$
\mathscr{P}_{3, d-1}(5)=\left\{\mu_{1}=(5,0,0), \mu_{2}=(4,1,0), \mu_{3}=(3,2,0), \mu_{4}=(3,1,1), \mu_{5}=(2,2,1)\right\} .
$$

Thus the basis polynomial $P$ can be written as

$$
P=\alpha_{1}\left[x_{1}^{6}\right]+\alpha_{2}\left[x_{1}^{5} x_{2}\right]+\alpha_{3}\left[x_{1}^{4} x_{2}^{2}\right]+\underset{8}{\alpha_{4}\left[x_{1}^{4} x_{2} x_{3}\right]+\alpha_{5}\left[x_{1}^{3} x_{2}^{3}\right]+\alpha_{6}\left[x_{1}^{3} x_{2}^{2} x_{3}\right]+\alpha_{7}\left[x_{1}^{2} x_{2}^{2} x_{3}^{2}\right] .}
$$


Using the same method as above, we obtain that

$$
\begin{array}{rlrl}
E\left(\left[x^{\lambda_{1}}\right]\right) & =6\left[x_{1}^{5}\right] & & C_{11}\left[x^{\mu_{1}}\right], \\
E\left(\left[x^{\lambda_{2}}\right]\right) & =12\left[x_{1}^{5}\right]+10\left[x_{1}^{4} x_{2}\right] & & C_{12}\left[x^{\mu_{1}}\right]+C_{22}\left[x^{\mu_{2}}\right], \\
E\left(\left[x^{\lambda_{3}}\right]\right) & =10\left[x_{1}^{4} x_{2}\right]+12\left[x_{1}^{3} x_{2}^{2}\right] & & C_{23}\left[x^{\mu_{2}}\right]+C_{33}\left[x^{\mu_{3}}\right], \\
E\left(\left[x^{\lambda_{4}}\right]\right) & =6\left[x_{1}^{4} x_{2}\right]+12\left[x_{1}^{3} x_{2} x_{3}\right] & =C_{24}\left[x^{\mu_{2}}\right]+C_{44}\left[x^{\mu_{4}}\right], \\
E\left(\left[x^{\lambda_{5}}\right]\right) & =12\left[x_{1}^{3} x_{2}^{2}\right] & =C_{35}\left[x^{\mu_{3}}\right], \\
E\left(\left[x^{\lambda_{6}}\right]\right) & =6\left[x_{1}^{3} x_{2}^{2}\right]+20\left[x_{1}^{3} x_{2} x_{3}\right]+24\left[x_{1}^{2} x_{2}^{2} x_{3}\right] & =C_{36}\left[x^{\mu_{3}}\right]+C_{46}\left[x^{\mu_{4}}\right]+C_{56}\left[x^{\mu_{5}}\right], \\
E\left(\left[x^{\lambda_{7}}\right]\right) & =10\left[x_{1}^{2} x_{2}^{2} x_{3}\right] & =C_{57}\left[x^{\mu_{5}}\right] .
\end{array}
$$

Thus

$$
C=\left(\begin{array}{ccccccc}
6 & 12 & 0 & 0 & 0 & 0 & 0 \\
0 & 10 & 10 & 6 & 0 & 0 & 0 \\
0 & 0 & 12 & 0 & 12 & 6 & 0 \\
0 & 0 & 0 & 12 & 0 & 20 & 0 \\
0 & 0 & 0 & 0 & 0 & 24 & 10
\end{array}\right)
$$

so we have the following linear system

$$
\alpha_{1}+2 \alpha_{2}=0, \quad 5 \alpha_{2}+5 \alpha_{3}+3 \alpha_{4}=0, \quad 2 \alpha_{3}+2 \alpha_{5}+\alpha_{6}=0, \quad 3 \alpha_{4}+5 \alpha_{6}=0, \quad 12 \alpha_{6}+5 \alpha_{7}=0 .
$$

Since the rank of $C$ is 5 , which is $p_{3,6}(5)$, there are two copies of trivial representation in $\operatorname{Ker}(E) \cap A(7)_{6}$ and we can find explicit basis polynomial of each of them by choosing appropriate values of $a_{5}$ and $a_{7}$. (Of course, they should be linearly independent.) One can easily verify that $\operatorname{triv}(7,6)=p_{3,6}(6)-p_{3,6}(5)=7-5=2$. Also we have $C=\left(C_{p q}\right)$ as follows:

$$
C=\left(\begin{array}{ccccccc}
C_{(5,0,0),(6,0,0)} & C_{(5,0,0),(5,1,0)} & 0 & 0 & 0 & 0 & 0 \\
0 & C_{(4,1,0),(5,1,0)} & C_{(4,1,0),(4,2,0)} & C_{(4,1,0),(4,1,1)} & 0 & 0 & 0 \\
0 & 0 & C_{(3,2,0),(4,2,0)} & 0 & C_{(3,2,0),(3,3,0)} & C_{(3,2,0),(3,2,1)} & 0 \\
0 & 0 & 0 & 0 & 0 & 0 & C_{(3,1,1,1),(3,2,1)} \\
0 & 0 & 0 & 0 & C_{(2,2,1),(3,2,1)} & C_{(2,2,1),(2,2,2)}
\end{array}\right) .
$$

Then the submatrices $C(a)$ are given as

$$
\begin{aligned}
C(5) & =\left(\begin{array}{llllllll}
C_{(5,0,0),(6,0,0)} & C_{(5,0,0),(5,1,0)} & 0 & 0 & 0 & 0 & 0
\end{array}\right), \\
C(4) & =\left(\begin{array}{llllllll}
0 & C_{(4,1,0),(5,1,0)} & C_{(4,1,0),(4,2,0)} & C_{(4,1,0),(4,1,1)} & 0 & 0 & 0
\end{array}\right), \\
C(3) & =\left(\begin{array}{llllllll}
0 & 0 & C_{(3,2,0),(4,2,0)} & 0 & C_{(3,2,0),(3,3,0)} & C_{(3,2,0),(3,2,1)} & 0 \\
0 & 0 & 0 & C_{(3,1,1),(4,1,1)} & 0 & C_{(3,1,1),(3,2,1)} & 0
\end{array}\right), \\
C(2) & =\left(\begin{array}{llllllll}
0 & 0 & 0 & 0 & 0 & C_{(2,2,1),(3,2,1)} & C_{(2,2,1),(2,2,2)}
\end{array}\right) .
\end{aligned}
$$

Note that each $C(a)$ is in row echelon form, and so its rank is equal to the number of rows in $C(a)$ for $a=5,4,3,2$. Hence the matrix $C$ is in block lower triangular form and we have

$$
\operatorname{rank} C=\sum_{a=2}^{5} \operatorname{rank} C(a)=\sum_{a=2}^{5}(\text { the number of rows in } C(a))=\text { the number of rows in } C .
$$

(c) Let $d=7, j=8$. Then we have

$$
\begin{aligned}
& \mathscr{P}_{3, d-1}(8)=\left\{\begin{array}{c}
\lambda_{1}=(6,2,0), \lambda_{2}=(6,1,1), \lambda_{3}=(5,3,0), \lambda_{4}=(5,2,1), \\
\lambda_{5}=(4,4,0), \lambda_{6}=(4,3,1), \lambda_{7}=(4,2,2), \lambda_{8}=(3,3,2)
\end{array}\right\}, \text { and } \\
& \mathscr{P}_{3, d-1}(7)=\left\{\begin{array}{c}
\mu_{1}=(6,1,0), \mu_{2}=(5,2,0), \mu_{3}=(5,1,1), \mu_{4}=(4,3,0), \\
\mu_{5}=(4,2,1), \mu_{6}=(3,3,1), \mu_{7}=(3,2,2) \\
9
\end{array}\right\} .
\end{aligned}
$$


Thus the basis polynomial $P$ can be written as

$$
P=\alpha_{1}\left[x_{1}^{6} x_{2}^{2}\right]+\alpha_{2}\left[x_{1}^{6} x_{2} x_{3}\right]+\alpha_{3}\left[x_{1}^{5} x_{2}^{3}\right]+\alpha_{4}\left[x_{1}^{5} x_{2}^{2} x_{3}\right]+\alpha_{5}\left[x_{1}^{4} x_{2}^{4}\right]+\alpha_{6}\left[x_{1}^{4} x_{2}^{3} x_{3}\right]+\alpha_{7}\left[x_{1}^{4} x_{2}^{2} x_{3}^{2}\right]+a_{8}\left[x_{1}^{3} x_{2}^{3} x_{3}^{2}\right] .
$$

By a simple calculation as above, we get that

$$
\begin{array}{rlrl}
E\left(\left[x^{\lambda_{1}}\right]\right) & =10\left[x^{\mu_{1}}\right]+6\left[x^{\mu_{2}}\right] & & =C_{11}\left[x^{\mu_{1}}\right]+C_{21}\left[x^{\mu_{2}}\right], \\
E\left(\left[x^{\lambda_{2}}\right]\right) & =6\left[x^{\mu_{1}}\right]+6\left[x^{\mu_{3}}\right] & & C_{12}\left[x^{\mu_{1}}\right]+C_{32}\left[x^{\mu_{3}}\right], \\
E\left(\left[x^{\lambda_{3}}\right]\right) & =12\left[x^{\mu_{2}}\right]+10\left[x^{\mu_{4}}\right] & =C_{23}\left[x^{\mu_{2}}\right]+C_{43}\left[x^{\mu_{4}}\right], \\
E\left(\left[x^{\lambda_{4}}\right]\right) & =6\left[x^{\mu_{2}}\right]+20\left[x^{\mu_{3}}\right]+10\left[x^{\mu_{5}}\right] & =C_{24}\left[x^{\mu_{2}}\right]+C_{34}\left[x^{\mu_{3}}\right]+C_{54}\left[x^{\mu_{5}}\right], \\
E\left(\left[x^{\lambda_{5}}\right]\right) & =12\left[x^{\mu_{4}}\right] & =C_{45}\left[x^{\mu_{4}}\right], \\
E\left(\left[x^{\lambda_{6}}\right]\right) & =6\left[x^{\mu_{4}}\right]+12\left[x^{\mu_{5}}\right]+12\left[x^{\mu_{6}}\right] & =C_{46}\left[x^{\mu_{4}}\right]+C_{56}\left[x^{\mu_{5}}\right]+C_{66}\left[x^{\mu_{6}}\right], \\
E\left(\left[x^{\lambda_{7}}\right]\right) & =10\left[x^{\mu_{5}}\right]+12\left[x^{\mu_{7}}\right] & =C_{57}\left[x^{\mu_{5}}\right]+C_{77}\left[x^{\mu_{7}}\right], \\
E\left(\left[x^{\lambda_{8}}\right]\right) & =10\left[x^{\mu_{6}}\right]+12\left[x^{\mu_{7}}\right] & =C_{68}\left[x^{\mu_{6}}\right]+C_{78}\left[x^{\mu_{8}}\right],
\end{array}
$$

so

$$
C=\left(\begin{array}{cccccccc}
10 & 6 & 0 & 0 & 0 & 0 & 0 & 0 \\
6 & 0 & 12 & 6 & 0 & 0 & 0 & 0 \\
0 & 6 & 0 & 20 & 0 & 0 & 0 & 0 \\
0 & 0 & 10 & 0 & 12 & 6 & 0 & 0 \\
0 & 0 & 0 & 10 & 0 & 12 & 10 & 0 \\
0 & 0 & 0 & 0 & 0 & 12 & 0 & 10 \\
0 & 0 & 0 & 0 & 0 & 0 & 12 & 12
\end{array}\right) .
$$

Since the rank of $C$ is 7 , which is $p_{3,6}(6)$, there is one copy of trivial representation in $\operatorname{Ker}(E) \cap A(7)_{8}$ and we can find explicit basis polynomial by choosing appropriate values of $a_{8}$. One can easily verify that $\operatorname{triv}(7,8)=p_{3,6}(8)-p_{3,6}(7)=8-7=1$. Also we have $C=\left(C_{p q}\right)$ as follows:

$C=\left(\begin{array}{ccccccc}C_{(6,1,0),(6,2,0)} & C_{(6,1,0),(6,1,1)} & 0 & 0 & 0 & 0 & 0 \\ C_{(5,2,0),(6,2,0)} & 0 & C_{(5,2,0),(5,3,0)} & C_{(5,2,0),(5,2,1)} & 0 & 0 & 0 \\ 0 & C_{(5,1,1),(6,1,1)} & 0 & C_{(5,1,1),(5,2,1)} & 0 & 0 \\ 0 & 0 & C_{(4,3,0),(5,3,0)} & 0 & C_{(4,3,0),(4,4,0)} & C_{(4,3,0),(4,3,1)} & C_{(4,2,1),(4,3,1)} C_{(4,2,1),(4,2,2)} \\ 0 & 0 & 0 & C_{(4,2,1),(5,2,1)} & 0 & 0 & 0 \\ 0 & 0 & 0 & 0 & 0 & C_{(3,3,1),(4,3,1)} & 0 \\ 0 & 0 & 0 & 0 & 0 & C_{(3,3,1),(3,3,2)}\end{array}\right)$

Then the submatrices $C(a)$ are given as

$$
\begin{aligned}
C(6) & =\left(\begin{array}{llllllll}
C_{(6,1,0),(6,2,0)} & C_{(6,1,0),(6,1,1)} & 0 & 0 & 0 & 0 & 0 & 0
\end{array}\right), \\
C(5) & =\left(\begin{array}{cccccccc}
C_{(5,2,0),(6,2,0)} & 0 & C_{(5,2,0),(5,3,0)} & C_{(5,2,0),(5,2,1)} & 0 & 0 & 0 & 0 \\
0 & C_{(5,1,1),(6,1,1)} & 0 & C_{(5,1,1),(5,2,1)} & 0 & 0 & 0 & 0
\end{array}\right), \\
C(4) & =\left(\begin{array}{lllllllll}
0 & 0 & C_{(4,3,0),(5,3,0)} & 0 & C_{(4,3,0),(4,4,0)} & C_{(4,3,0),(4,3,1)} & 0 & 0 \\
0 & 0 & 0 & C_{(4,2,1),(5,2,1)} & 0 & C_{(4,2,1),(4,3,1)} & C_{(4,2,1),(4,2,2)} & 0
\end{array}\right), \\
C(3) & =\left(\begin{array}{llllllllll}
0 & 0 & 0 & 0 & 0 & C_{(3,3,1),(4,3,1)} & 0 & C_{(3,3,1),(3,3,2)} \\
0 & 0 & 0 & 0 & 0 & 0 & C_{(3,2,2),(4,2,2)} & C_{(3,2,2),(3,3,2)}
\end{array}\right) .
\end{aligned}
$$

Note that each $C(a)$ is in row echelon form and its rank is equal to the number of rows in $C(a)$ for $a=6,5,4,3$. Hence the matrix $C$ is in block lower triangular form and we have

$$
\operatorname{rank} C=\sum_{a=3}^{6} \operatorname{rank} C(a)=\sum_{a=3}^{6}(\text { the number of rows in } C(a))=\text { the number of rows in } C .
$$


(d) Let $d=9, j=9$. Then we have

$$
\begin{aligned}
& \mathscr{P}_{3, d-1}(9)=\left\{\begin{array}{c}
\lambda_{1}=(8,1,0), \lambda_{2}=(7,2,0), \lambda_{3}=(7,1,1), \lambda_{4}=(6,3,0), \lambda_{5}=(6,2,1), \lambda_{6}=(5,4,0), \\
\lambda_{7}=(5,3,1), \lambda_{8}=(5,2,2), \lambda_{9}=(4,4,1), \lambda_{10}=(4,3,2), \lambda_{11}=(3,3,3)
\end{array}\right\} \quad \text { and } \\
& \mathscr{P}_{3, d-1}(8)=\left\{\begin{array}{l}
\mu_{1}=(8,0,0), \mu_{2}=(7,1,0), \mu_{3}=(6,2,0), \mu_{4}=(6,1,1), \mu_{5}=(5,3,0), \\
\mu_{6}=(5,2,1), \mu_{7}=(4,4,0), \mu_{8}=(4,3,1), \mu_{9}=(4,2,2), \mu_{10}=(3,3,2)
\end{array}\right\} .
\end{aligned}
$$

Thus the basis polynomial $P$ can be written as

$$
\begin{aligned}
P= & \alpha_{1}\left[x_{1}^{8} x_{2}\right]+\alpha_{2}\left[x_{1}^{7} x_{2}^{2}\right]+\alpha_{3}\left[x_{1}^{7} x_{2} x_{3}\right]+\alpha_{4}\left[x_{1}^{6} x_{2}^{3}\right]+\alpha_{5}\left[x_{1}^{6} x_{2}^{2} x_{3}\right]+\alpha_{6}\left[x_{1}^{5} x_{2}^{4}\right]+\alpha_{7}\left[x_{1}^{5} x_{2}^{3} x_{3}^{2}\right]+ \\
& \alpha_{8}\left[x_{1}^{4} x_{2}^{4} x_{3}\right]+\alpha_{9}\left[x_{1}^{4} x_{2}^{3} x_{3}^{2}\right]+\alpha_{10}\left[x_{1}^{4} x_{2}^{3} x_{3}^{2}\right]+\alpha_{11}\left[x_{1}^{4} x_{2}^{3} x_{3}^{2}\right] .
\end{aligned}
$$

By a simple calculation as above, we get that

$$
\begin{aligned}
& E\left(\left[x^{\lambda_{1}}\right]\right)=16\left[x^{\mu_{1}}\right]+8\left[x^{\mu_{2}}\right] \quad=C_{11}\left[x^{\mu_{1}}\right]+C_{21}\left[x^{\mu_{2}}\right] \\
& E\left(\left[x^{\lambda_{2}}\right]\right)=14\left[x^{\mu_{2}}\right]+14\left[x^{\mu_{3}}\right] \quad=C_{22}\left[x^{\mu_{2}}\right]+C_{32}\left[x^{\mu_{3}}\right] \\
& E\left(\left[x^{\lambda_{3}}\right]\right)=8\left[x^{\mu_{2}}\right]+14\left[x^{\mu_{4}}\right] \quad=C_{23}\left[x^{\mu_{2}}\right]+C_{43}\left[x^{\mu_{4}}\right], \\
& E\left(\left[x^{\lambda_{4}}\right]\right)=18\left[x^{\mu_{3}}\right]+18\left[x^{\mu_{5}}\right] \quad=C_{34}\left[x^{\mu_{3}}\right]+C_{54}\left[x^{\mu_{4}}\right] \\
& E\left(\left[x^{\lambda_{5}}\right]\right)=8\left[x^{\mu_{3}}\right]+28\left[x^{\mu_{4}}\right]+18\left[x^{\mu_{6}}\right]=C_{45}\left[x^{\mu_{4}}\right] \\
& E\left(\left[x^{\lambda_{6}}\right]\right)=20\left[x^{\mu_{5}}\right]+20\left[x^{\mu_{7}}\right] \quad=C_{56}\left[x^{\mu_{5}}\right]+C_{76}\left[x^{\mu_{7}}\right], \\
& E\left(\left[x^{\lambda_{7}}\right]\right)=8\left[x^{\mu_{5}}\right]+18\left[x^{\mu_{6}}\right]+20\left[x^{\mu_{8}}\right]=C_{57}\left[x^{\mu_{5}}\right]+C_{67}\left[x^{\mu_{6}}\right]+C_{87}\left[x^{\mu_{8}}\right], \\
& E\left(\left[x^{\lambda_{8}}\right]\right)=14\left[x^{\mu_{6}}\right]+20\left[x^{\mu_{9}}\right] \quad=C_{68}\left[x^{\mu_{6}}\right]+C_{98}\left[x^{\mu_{9}}\right] \\
& E\left(\left[x^{\lambda_{9}}\right]\right)=8\left[x^{\mu_{7}}\right]+16\left[x^{\mu_{8}}\right]=C_{79}\left[x^{\mu_{8}}\right]+C_{89}\left[x^{\mu_{8}}\right]+C_{8,10} \text {, } \\
& E\left(\left[x^{\lambda_{10}}\right]\right)=14\left[x_{\mu_{8}}\right]+36\left[x^{\mu_{9}}\right]+20\left[x^{\mu_{10}}\right]=C_{8,10}\left[x^{\mu_{8}}\right]+C_{9,10}\left[x^{\mu_{9}}\right]+C_{10,10}\left[x^{\mu_{10}}\right], \\
& E\left(\left[x^{\lambda_{11}}\right]\right)=18\left[x^{\mu_{10}}\right] \quad=C_{10,11}\left[x^{\mu_{10}}\right] \text {, }
\end{aligned}
$$

so

$$
C=\left(\begin{array}{ccccccccccc}
16 & 0 & 0 & 0 & 0 & 0 & 0 & 0 & 0 & 0 & 0 \\
8 & 14 & 8 & 0 & 0 & 0 & 0 & 0 & 0 & 0 & 0 \\
0 & 14 & 0 & 18 & 8 & 0 & 0 & 0 & 0 & 0 & 0 \\
0 & 0 & 14 & 0 & 28 & 0 & 0 & 0 & 0 & 0 & 0 \\
0 & 0 & 0 & 18 & 0 & 20 & 8 & 0 & 0 & 0 & 0 \\
0 & 0 & 0 & 0 & 18 & 0 & 18 & 14 & 0 & 0 & 0 \\
0 & 0 & 0 & 0 & 0 & 20 & 0 & 0 & 8 & 0 & 0 \\
0 & 0 & 0 & 0 & 0 & 0 & 20 & 0 & 16 & 14 & 0 \\
0 & 0 & 0 & 0 & 0 & 0 & 0 & 20 & 0 & 36 & 0 \\
0 & 0 & 0 & 0 & 0 & 0 & 0 & 0 & 0 & 20 & 18
\end{array}\right)
$$

Since the rank of $C$ is 10 , which is $p_{3,8}(8)$, there is one copy of trivial representation in $\operatorname{Ker}(E) \cap A(9)_{9}$ and we can find explicit basis polynomial by choosing appropriate values of $a_{11}$. One can easily verify that $\operatorname{triv}(9,9)=p_{3,8}(9)-p_{3,8}(8)=11-10=1$. Then the submatrices $C(a)$ are given as

$$
\begin{aligned}
& C(8)=\left(\begin{array}{lllllllllll}
C_{(8,0,0),(8,1,0)} & 0 & 0 & 0 & 0 & 0 & 0 & 0 & 0 & 0 & 0
\end{array}\right),
\end{aligned}
$$

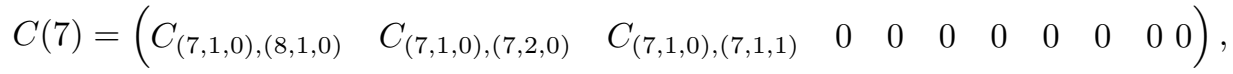

$$
\begin{aligned}
& C(6)=\left(\begin{array}{ccccccccccc}
0 & C_{(6,2,0),(7,2,0)} & 0 & C_{(6,2,0),(6,3,0)} & C_{(6,2,0),(6,2,1)} & 0 & 0 & 0 & 0 & 0 & 0 \\
0 & 0 & C_{(6,1,1),(7,1,1)} & 0 & C_{(6,1,1),(6,2,1)} & 0 & 0 & 0 & 0 & 0 & 0
\end{array}\right),
\end{aligned}
$$

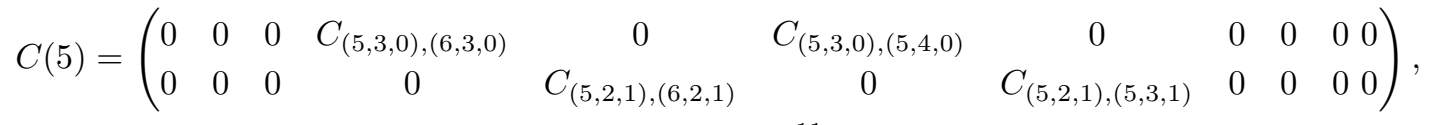




$$
\begin{aligned}
& C(4)=\left(\begin{array}{cccccccccc}
0 & 0 & 0 & 0 & 0 & C_{(4,4,0),(5,4,0)} & 0 & 0 & C_{(4,4,0),(4,4,1)} & 00 \\
0 & 0 & 0 & 0 & 0 & 0 & C_{(4,3,1),(5,3,1)} & 0 & C_{(4,3,1),(4,4,1)} & C_{(4,3,1),(4,3,2)} 0 \\
0 & 0 & 0 & 0 & 0 & 0 & 0 & C_{(4,2,2),(5,2,2)} & 0 & C_{(4,2,2),(4,3,2)}
\end{array}\right), \\
& C(3)=\left(\begin{array}{ccccccccccc}
0 & 0 & 0 & 0 & 0 & 0 & 0 & 0 & 0 & C_{(3,3,2),(4,3,2)} & C_{(3,3,2),(3,3,3)}
\end{array}\right) .
\end{aligned}
$$

Note that each $C(a)$ is in row echelon form and its rank is equal to the number of rows in $C(a)$ for $a=$ $8,7,6,5,4,3$. Hence the matrix $C$ is in block lower triangular form and we have

$$
\left.\operatorname{rank} C=\sum_{a=3}^{8} \operatorname{rank} C(a)=\sum_{a=3}^{8} \text { (the number of rows in } C(a)\right)=\text { the number of rows in } C \text {. }
$$

Lemma 3.4. With notations as above, for $d>j>0, C$ is a matrix with row echelon form and the rank of $C$ is the number of rows of $C$, which is $p_{3, d-1}(j-1)$.

Proof. Let

$$
\begin{aligned}
\mathscr{P}_{3, d-1}(j) & =\left\{\lambda_{1}=(j, 0,0), \lambda_{2}=(j-1,1,0), \lambda_{3}=(j-2,2,0), \lambda_{4}=(j-2,1,1), \ldots\right\}, \\
\mathscr{P}_{3, d-1}(j-1) & =\left\{\mu_{1}=(j-1,0,0), \mu_{2}=(j-2,1,0), \mu_{3}=(j-3,2,0), \mu_{4}=(j-3,1,1), \ldots\right\} .
\end{aligned}
$$

Note that

$$
\begin{aligned}
& E\left(\left[x^{\lambda_{1}}\right]\right)=j(d-j)\left[x^{\mu_{1}}\right] \quad=C_{11}\left[x^{\mu_{1}}\right], \\
& E\left(\left[x^{\lambda_{2}}\right]\right)=(d-1)\left[x^{\mu_{1}}\right]+(j-1)(d-j+1)\left[x^{\mu_{2}}\right]=C_{12}\left[x^{\mu_{1}}\right]+C_{22}\left[x^{\mu_{2}}\right] \text {, } \\
& E\left(\left[x^{\lambda_{3}}\right]\right)=2(d-2)\left[x^{\mu_{2}}\right]+(j-2)(d-j+2)\left[x^{\mu_{3}}\right]=C_{23}\left[x^{\mu_{2}}\right]+C_{33}\left[x^{\mu_{3}}\right] \text {, } \\
& E\left(\left[x^{\lambda_{4}}\right]\right)=(d-1)\left[x^{\mu_{2}}\right]+(j-2)(d-j+2)\left[x^{\mu_{4}}\right]=C_{24}\left[x^{\mu_{2}}\right]+C_{44}\left[x^{\mu_{4}}\right] \text {, }
\end{aligned}
$$

So $C$ is of the form

$$
C=\left(\begin{array}{cccccc}
C_{11} & C_{12} & 0 & 0 & * & \cdots \\
0 & C_{22} & C_{23} & C_{24} & * & \cdots \\
0 & 0 & C_{33} & 0 & * & \cdots \\
0 & 0 & 0 & C_{44} & * & \cdots \\
0 & 0 & 0 & 0 & * & \cdots \\
\vdots & \vdots & \vdots & \vdots & \vdots & \vdots
\end{array}\right)
$$

Claim. If $C_{i, \ell} \neq 0$ and $C_{k, \ell}=0$ for $k>i$, then $C_{k+1, \ell+1}=0$ for such $k$.

Proof of Claim. Let $\mu_{k}=(a, b, c)$ such that $a+b+c=j-1$ and $j-1 \geq a \geq b \geq c$. Since $C_{k, j}=0$, we see that no $\lambda_{j}$ is

$$
(a+1, b, c),(a, b+1, c),(a, b, c+1) .
$$

Note that

$$
\mu_{k+1}=(a, b-1, c+1),(a-1, j-a, 0) .
$$

If $C_{k+1, j+1} \neq 0$, then $\lambda_{j+1}$ is one of the following,

$$
(a+1, b-1, c+1),(a, b, c+1),(a, b-1, c+2),
$$

which is impossible. Hence $C_{k+1, j+1}=0$ for such $k$.

It is from Claim that the matrix $C$ is a row echelon form. Moreover, since $E\left(\left[x^{\lambda_{i}}\right]\right)$ generates all partitions in $\mathscr{P}_{3, d-1}(j-1)$, we see that every row of $C$ has non zero component, i.e., the rank of $C$ is the same as the number of rows of $C$, which $p_{3, d-1}(j-1)$, as we wished.

Lemma 3.5. With notations as above, for $d \leq j$, the rank of $C$ is the number of rows of $C$, which is $p_{3, d-1}(j-1)$. 
Proof. Let

$$
\begin{aligned}
\mathscr{P}_{3, d-1}(j) & =\left\{\lambda_{1}=(j-1,1,0), \lambda_{2}=(j-2,2,0), \lambda_{3}=(j-2,1,1), \lambda_{4}=(j-3,3,0), \ldots\right\}, \\
\mathscr{P}_{3, d-1}(j-1) & =\left\{\mu_{1}=(j-1,0,0), \mu_{2}=(j-2,1,0), \mu_{3}=(j-3,2,0), \mu_{4}=(j-3,1,1), \ldots\right\} .
\end{aligned}
$$

Note that

$$
\begin{aligned}
& E\left(\left[x^{\lambda_{1}}\right]\right)=2(d-1)\left[x^{\mu_{1}}\right]+(j-1)(d-j+1)\left[x^{\mu_{2}}\right]=C_{11}\left[x^{\mu_{1}}\right]+C_{21}\left[x^{\mu_{2}}\right], \\
& E\left(\left[x^{\lambda_{2}}\right]\right)=2(d-2)\left[x^{\mu_{2}}\right]+(j-2)(d-j+2)\left[x^{\mu_{3}}\right]=C_{22}\left[x^{\mu_{2}}\right]+C_{32}\left[x^{\mu_{3}}\right], \\
& E\left(\left[x^{\lambda_{3}}\right]\right)=(d-1)\left[x^{\mu_{2}}\right]+(j-2)(d-j+2)\left[x^{\mu_{4}}\right]=C_{33}\left[x^{\mu_{2}}\right]+C_{43}\left[x^{\mu_{4}}\right], \\
& E\left(\left[x^{\lambda_{4}}\right]\right)=3(d-3)\left[x^{\mu_{3}}\right]+(j-3)(d-j+3)\left[x^{\mu_{5}}\right]=C_{34}\left[x^{\mu_{3}}\right]+C_{54}\left[x^{\mu_{5}}\right],
\end{aligned}
$$

So $C$ is of the form

$$
C=\left(\begin{array}{cccccc}
C_{11} & 0 & 0 & 0 & * & \cdots \\
C_{21} & C_{22} & 0 & 0 & * & \cdots \\
0 & C_{32} & C_{33} & C_{34} & * & \cdots \\
0 & 0 & C_{43} & 0 & * & \cdots \\
0 & 0 & 0 & C_{54} & * & \cdots \\
\vdots & \vdots & \vdots & \vdots & \vdots & \vdots
\end{array}\right)
$$

Claim. If $C_{i, \ell} \neq 0$ and $C_{k, \ell}=0$ for $k>i \geq 2$, then $C_{k+1, \ell+1}=0$ for such $k$.

Proof of Claim. Let $\mu_{k}=(a, b, c)$ such that $a+b+c=j-1$ and $j-1 \geq a \geq b \geq c$. Since $C_{k, j}=0$, we see that no $\lambda_{j}$ is

$$
(a+1, b, c),(a, b+1, c),(a, b, c+1) .
$$

Note that

$$
\mu_{k+1}=(a, b-1, c+1),(a-1, j-a, 0) .
$$

If $C_{k+1, j+1} \neq 0$, then $\lambda_{j+1}$ is one of the following,

$$
(a+1, b-1, c+1),(a, b, c+1),(a, b-1, c+2),
$$

which is impossible. Hence $C_{k+1, j+1}=0$ for such $k$.

It is from Claim that the matrix $C$ is a row echelon form. Moreover, since $E\left(\left[x^{\lambda_{i}}\right]\right)$ generates all partitions in $\mathscr{P}_{3, d-1}(j-1)$, we see that every row of $C$ has non zero component, i.e., the rank of $C$ is the same as the number of rows of $C$, which $p_{3, d-1}(j-1)$, as we wished.

Theorem 3.6. Given the exponent $d$ and the degree $j$, let

$$
P=\sum_{\lambda=(a, b, c)} \alpha_{a, b, c}\left[x_{1}^{a} x_{2}^{b} x_{3}^{c}\right]
$$

be a homogeneous symmetric polynomial of degree $j$, where $\lambda=(a, b, c)$ runs over all partitions of $j$ of size $3 \times(d-1)$ and $\left[x_{1}^{a} x_{2}^{b} x_{3}^{c}\right]$ denotes the $S_{3}$-orbit of $x_{1}^{a} x_{2}^{b} x_{3}^{c}$.

(a) The solutions of the system of linear equations $E(P)=0$ give explicit basis polynomials for each trivial representation in $\operatorname{Ker}(E) \cap A(d)_{j}$.

(b) Let $\operatorname{triv}(d, j)$ denote the multiplicity of trivial representation in the irreducible decomposition of $\operatorname{Ker}(E) \cap A(d)_{j}$. Then

$$
\operatorname{triv}(d, j)=p_{3, d-1}(j)-p_{3, d-1}(j-1)
$$


Proof. The statement (a) is proved in the discussion above the theorem.

To prove (b), let $\lambda_{1}, \ldots, \lambda_{t}$ be the partitions in $\mathscr{P}_{3, d-1}(j)$ and let $\mu_{1}, \ldots, \mu_{s}$ be the partitions in $\mathscr{P}_{3, d-1}(j-$ 1) listed by the lexicographic ordering. Thus $t=p_{3, d-1}(j)$ and $s=p_{3, d-1}(j-1)$.

For $\lambda=(a, b, c)$, we denote $x^{\lambda}=x_{1}^{a} x_{2}^{b} x_{3}^{c}$ and write $P=\alpha_{1}\left[x^{\lambda_{1}}\right]+\cdots+\alpha_{t}\left[x^{\lambda_{t}}\right]$ be a homogeneous symmetric polynomial of degree $j$. Then the condition $E(P)=0$ yields a system of linear equations in the variable $\alpha_{1}, \ldots, \alpha_{t}$ with coefficient matrix $C=\left(C_{p q}\right)$ for $(1 \leq p \leq s, 1 \leq q \leq t)$, where $C_{p q}:=C_{\mu_{p}, \lambda_{q}}$ such that

$$
E\left(\alpha_{p}\left[x^{\lambda_{q}}\right]\right)=\alpha_{p} C_{\mu_{p}, \lambda_{q}}\left[x^{\mu_{p}}\right] .
$$

That is,

$$
\begin{gathered}
C_{11} \alpha_{1}+\cdots+C_{1 t} \alpha_{t}=0 \\
C_{21} \alpha_{1}+\cdots+C_{2 t} \alpha_{t}=0 \\
\vdots \\
C_{s 1} \alpha_{1}+\cdots+C_{s t} \alpha_{t}=0
\end{gathered}
$$

Observe that the coefficients $C_{p q}=0$ unless $\lambda_{q}=\mu_{p}+(1,0,0), \lambda_{q}=\mu_{p}+(0,1,0)$, or $\lambda_{q}=\mu_{p}+(0,0,1)$ and they are partitions. In other words, $C_{p q}=0$ unless the term $\left[x^{\mu_{p}}\right]$ arises as an image of $\left[x^{\mu_{p}+(1,0,0)}\right]$, $\left[x^{\mu_{p}+(0,1,0)}\right]$, or $\left[x^{\mu_{p}+(0,0,1)}\right]$ under $E$. Also note that at least one of them is a partition. (See Lemma 3.4, 3.5.)

Let $C(a)$ be the submatrix of $C$ such that i) the non-zero entries are determined by the partitions $\mu \in$ $\mathscr{P}_{3, d-1}(j-1), \lambda \in \mathscr{P}_{3, d-1}(j)$, where the first component of $\mu$ is $a$ and the first component of $\lambda$ is $a$ or $a+1$, ii) the other entries are 0. (See Example 3.3 above.) Then each $C(a)$ is in row echelon form. Since $C_{(a, b, c),\left(a^{\prime}, b^{\prime}, c^{\prime}\right)}=0$ unless $a^{\prime}=a$ or $a^{\prime}=a+1$, the matrix $C$ is in block low triangular form and hence we have

$$
\operatorname{rank} C=\sum_{a} \operatorname{rank} C(a)=\sum_{a}(\text { the number of rows in } C(a))=\text { the number of rows in } C,
$$

which proves our claim.

\section{(2) Sign representation:}

Given $d$ and $j$, since every permutaion acts as the multiplication by its sign, the candidate polynomial in $\operatorname{Ker}(E) \cap A(d)_{j}$ can be written as

$$
P=\sum_{\mu=(a, b, c)} \beta_{a, b, c}\left\langle x_{1}^{a} x_{2}^{b} x_{3}^{c}\right\rangle
$$

where $\mu=(a, b, c)$ is a strict partition of $j$ of size $3 \times(d-1)$ and $\left\langle x_{1}^{a} x_{2}^{b} x_{3}^{c}\right\rangle$ denotes the $S_{3}$-orbit of the monomial $x_{1}^{a} x_{2}^{b} x_{3}^{c}$ (under the sign action). For instance, when $d \geq 4$, we have

$$
\begin{aligned}
& \left\langle x_{1}^{3}\right\rangle=0, \\
& \left\langle x_{1}^{2} x_{2}\right\rangle=x_{1}^{2} x_{2}-x_{1}^{2} x_{3}+x_{2}^{2} x_{3}-x_{1} x_{2}^{2}+x_{1} x_{3}^{2}-x_{2} x_{3}^{2}, \\
& \left\langle x_{1} x_{2} x_{3}\right\rangle=0 .
\end{aligned}
$$

As is the case of trivial representation, the solutions of the system $E(P)=0$ would give the explicit basis polynomials of each sign representation in $\operatorname{Ker}(E) \cap A(d)_{j}$ and their multipicity is given by $p_{3, d-1}^{+}(j)-$ $p_{3, d-1}^{+}(j-1)$, where $p_{k, l}^{+}(n)$ denotes the number of strict partitions of size $k \times l$. Hence we have:

Theorem 3.7. Given the exponent $d$ and the degree $j$, let

$$
P=\sum_{\mu=(a, b, c)} \beta_{a, b, c}\left\langle x_{1}^{a} x_{2}^{b} x_{3}^{c}\right\rangle
$$


be a homogeneous skew-symmetric polynomial of degree $j$, where $\mu=(a, b, c)$ runs over all rectangular strict partitions of $j$ of size $3 \times(d-1)$ and $\left\langle x_{1}^{a} x_{2}^{b} x_{3}^{c}\right\rangle$ denotes the $S_{3}$-orbit of $x_{1}^{a} x_{2}^{b} x_{3}^{c}$ under the sign action.

(a) The solutions of the system of linear equations $E(P)=0$ give explicit basis polynomials for each sign representation in $\operatorname{Ker}(E) \cap A(d)_{j}$.

(b) Let $\operatorname{sign}(d, j)$ denote the multiplicity of sign representation in the irreducible decomposition of $\operatorname{Ker}(E) \cap$ $A(d)_{j}$. Then

$$
\operatorname{sign}(d, j)=p_{3, d-1}^{+}(j)-p_{3, d-1}^{+}(j-1) .
$$

Proof. The statement (a) is proved in the discussion above the theorem. The statement (b) can be proved using a similar argument in Theorem 3.1(b).

\section{Example 3.8.}

(a) Let $d=5, j=4$. Then $\mu=(3,1,0)$ is the only strict partition of 4 of size $3 \times 4$ and hence the only candidate polynomial is

$$
P=x_{1}^{3} x_{2}-x_{1}^{3} x_{3}+x_{2}^{3} x_{3}-x_{1} x_{2}^{3}+x_{1} x_{3}^{3}-x_{2} x_{3}^{3} .
$$

But one can check $E(P) \neq 0$, which implies there is no sign representation in $\operatorname{Ker}(E) \cap A(5)_{4}$.

(b) Let $d=9, j=9$. Then we have

$\mathscr{P}_{3, d-1}^{+}(9)=\left\{\lambda_{1}=(8,1,0), \lambda_{2}=(7,2,0), \lambda_{3}=(6,3,0), \lambda_{4}=(6,2,1), \lambda_{5}=(5,4,0), \lambda_{6}=(5,3,1), \lambda_{7}=(4,3,2)\right\}$

and

$$
\mathscr{P}_{3, d-1}^{+}(8)=\left\{\mu_{1}=(7,1,0), \mu_{2}=(6,2,0), \mu_{3}=(5,3,0), \mu_{4}=(5,2,1), \mu_{5}=(4,3,1)\right\} .
$$

Thus the basis polynomial $P$ can be written as

$$
P=a_{1}\left\langle x_{1}^{8} x_{2}\right\rangle+a_{2}\left\langle x_{1}^{7} x_{2}^{2}\right\rangle+a_{3}\left\langle x_{1}^{6} x_{2}^{3}\right\rangle+a_{4}\left\langle x_{1}^{6} x_{2}^{2} x_{3}\right\rangle+a_{5}\left\langle x_{1}^{5} x_{2}^{4}\right\rangle+a_{6}\left\langle x_{1}^{5} x_{2}^{3} x_{3}\right\rangle+a_{7}\left\langle x_{1}^{4} x_{2}^{3} x_{3}^{2}\right\rangle .
$$

The condition $E(P)=0$ yields

$$
4 a_{1}+7 a_{2}=0, \quad 7 a_{2}+9 a_{3}+4 a_{4}=0, \quad 9 a_{3}+10 a_{5}+4 a_{6}=0, \quad a_{4}+a_{6}=0, \quad 10 a_{6}+7 a_{7}=0,
$$

and we obtain

$$
a_{1}=-\frac{5}{2} a_{5}-2 a_{6}, \quad a_{2}=\frac{10}{7} a_{5}+\frac{8}{7} a_{6}, \quad a_{3}=-\frac{10}{9} a_{5}-\frac{4}{9} a_{6}, \quad a_{4}=-a_{6}, \quad a_{7}=-\frac{10}{7} a_{6} .
$$

Hence there are two copies of sign representation in $\operatorname{Ker}(E) \cap A(9)_{9}$ and we can easily find their explicit bases. Note that $\operatorname{sign}(9,9)=p_{3,8}^{+}(9)-p_{3,8}^{+}(8)=7-5=2$ as expected.

Also we have $C=\left(C_{p q}\right)$ as follows:

$$
\begin{aligned}
& C=\left(\begin{array}{lllllll}
C_{11} & C_{12} & C_{13} & C_{14} & C_{15} & C_{16} & C_{17} \\
C_{21} & C_{22} & C_{23} & C_{24} & C_{25} & C_{26} & C_{27} \\
C_{31} & C_{32} & C_{33} & C_{34} & C_{35} & C_{36} & C_{37} \\
C_{41} & C_{42} & C_{43} & C_{44} & C_{45} & C_{46} & C_{47} \\
C_{51} & C_{52} & C_{53} & C_{54} & C_{55} & C_{56} & C_{57}
\end{array}\right)
\end{aligned}
$$

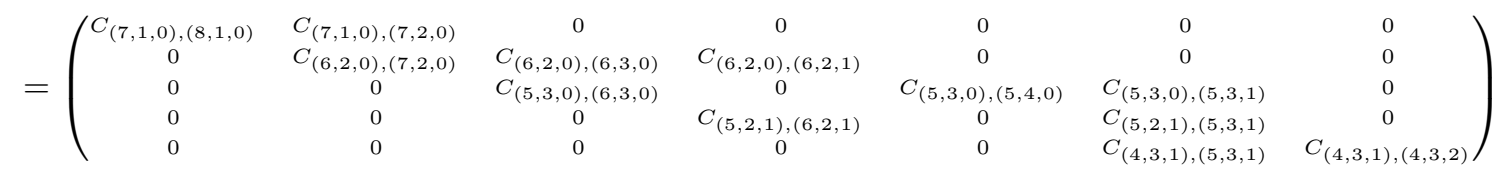

Then the submatrices $C(a)$ are given as

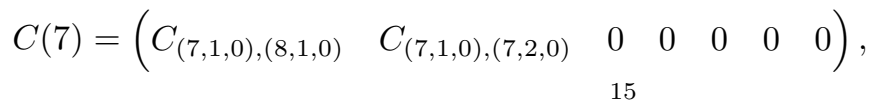




$$
\begin{aligned}
C(6) & =\left(\begin{array}{lllllll}
0 & C_{(6,2,0),(7,2,0)} & C_{(6,2,0),(6,3,0)} & C_{(6,2,0),(6,2,1)} & 0 & 0 & 0
\end{array}\right), \\
C(5) & =\left(\begin{array}{ccccccc}
0 & 0 & C_{(5,3,0),(6,3,0)} & 0 & C_{(5,3,0),(5,4,0)} & C_{(5,3,0),(5,3,1)} & 0 \\
0 & 0 & 0 & C_{(5,2,1),(6,2,1)} & 0 & C_{(5,2,1),(5,3,1)} & 0
\end{array}\right), \\
C(4) & =\left(\begin{array}{llllllll}
0 & 0 & 0 & 0 & 0 & C_{(4,3,1),(5,3,1)} & C_{(4,3,1),(4,3,2)}
\end{array}\right)
\end{aligned}
$$

Note that each $C(a)$ is in row echelon form and its rank is equal to the number of rows in $C(a)$ for $a=6,5,4,3$. Hence the matrix $C$ is in block lower triangular form and we have

$$
\operatorname{rank} C=\sum_{a=4}^{7} \operatorname{rank} C(a)=\sum_{a=4}^{7}(\text { the number of rows in } C(a))=\text { the number of rows in } C .
$$

\section{(3) Standard representation:}

Recall that the standard representation is the Specht module corresponding to the partition $\lambda=(2,1,0)$ and that it can be realized as the 2-dimensional hyperplane $H=\left\{\left(x_{1}, x_{2}, x_{3}\right) \in \mathbb{k}^{3} \mid x_{1}+x_{2}+x_{3}=0\right\}$. We can take $u_{1}=(1,-1,0)$ and $u_{2}=(0,1,-1)$ as the basis vectors for $H$ and if we set $u_{3}=(-1,0,1)$, we have $u_{1}+u_{2}+u_{3}=(0,0,0)$. Note that $(1,2) u_{1}=-u_{1}, u_{2}=-(1,3) u_{1}$ and $u_{3}=-(1,2) u_{2}$, where $(i, j)$ denotes he transposition of $i$ and $j$. This observation is the beginning point of our construction.

Given $d$ and $j$, let $P_{1}$ be a homogeneous polynomial of degree $j$ such that $(1,2) P_{1}=-P_{1}$. Set $P_{2}=-(1,3) P_{1}$ and $P_{3}=-(1,2) P_{2}$. Since the symmetric group action commutes with $E$ and $F, E\left(P_{1}\right)=0$ implies $E\left(P_{2}\right)=$ $E\left(P_{3}\right)=0$. We naturally take the system of linear equations arising form $E\left(P_{1}\right)=0$ and $P_{1}+P_{2}+P_{3}=0$. Then the solutions of this system would give the explicit basis polynomials of each standard representation in $\operatorname{Ker}(E) \cap A(d)_{j}$. Moreover, it is straightforward to see that the multiplicity of the standard representation in $\operatorname{Ker}(E) \cap A(d)_{j}$ is determined by those of trivial representation and sign representation (and the Hilbert polynomial).

To summarize, we have:

Theorem 3.9. Given the exponent $d$ and the degree $j$, let $P_{1}$ be a homogeneous polynomial of degree $j$ such that $(1,2) P_{1}=-P_{1}$. Set $P_{2}=-(1,3) P_{1}$ and $P_{3}=-(1,2) P_{2}$.

(a) The solutions of the system of linear equations $E\left(P_{1}\right)=0$ and $P_{1}+P_{2}+P_{3}=0$ give explicit basis polynomials $P_{1}$ and $P_{2}$ for each standard representation in $\operatorname{Ker}(E) \cap A(d)_{j}$.

(b) Let $\operatorname{st}(d, j)$ denote the multiplicity of standard representation in the irreducible decomposition of $\operatorname{Ker}(E) \cap A(d)_{j}$. Then

$$
\operatorname{st}(d, j)=\frac{1}{2}(\operatorname{mult}(d, j)-\operatorname{triv}(d, j)-\operatorname{sign}(d, j)),
$$

where mult $(d, j), \operatorname{triv}(d, j)$ and $\operatorname{sign}(d, j)$ are given in (3.2), (3.4) and (3.5), respectively.

\section{Example 3.10.}

(a) Let $d=3, j=3$. Set

$$
P_{1}=a\left(x_{1}^{2}-x_{1}^{2}\right)+b\left(x_{1}^{2} x_{3}-x_{2}^{2} x_{3}\right)+c\left(x_{1} x_{3}^{2}-x_{2} x_{3}^{2}\right),
$$

which satisfies the condition $(1,2) P_{1}=-P_{1}$. The condition $E\left(P_{1}\right)=0$ yields

$$
a+b=0, \quad b+c=0 .
$$

Thus we may take

$$
P_{1}=x_{1}^{2} x_{2}-x_{1} x_{2}^{2}-x_{1}^{2} x_{3}+x_{2}^{2} x_{3}+x_{1} x_{3}^{2}-x_{2} x_{3}^{2},
$$


from which we obtain

$$
\begin{aligned}
& P_{2}=x_{2}^{2} x_{3}-x_{2} x_{3}^{2}+x_{1} x_{3}^{2}-x_{1} x_{2}^{2}-x_{1}^{2} x_{3}+x_{1}^{2} x_{2}, \\
& P_{3}=x_{1} x_{3}^{2}-x_{1}^{2} x_{3}-x_{2} x_{3}^{2}+x_{1}^{2} x_{2}+x_{2}^{2} x_{3}-x_{1} x_{2}^{2} .
\end{aligned}
$$

But $P_{1}+P_{2}+P_{3} \neq 0$ and hence there is no standard representation in $\operatorname{Ker}(E) \cap A(3)_{3}$.

(b) Let $d=5, j=4$. Using the condition $(1,2) P_{1}=-P_{1}$, we may write

$$
\begin{aligned}
P_{1}= & a\left(x_{1}^{4}-x_{2}^{4}\right)+b\left(x_{1}^{3} x_{2}-x_{1} x_{2}^{3}\right)+c\left(x_{1}^{3} x_{3}-x_{2}^{3} x_{3}\right)+d\left(x_{1}^{2} x_{3}^{2}-x_{2}^{2} x_{3}^{2}\right) \\
& +e\left(x_{1} x_{3}^{3}-x_{2} x_{3}^{3}\right)+f\left(x_{1}^{2} x_{2} x_{3}-x_{1} x_{2}^{2} x_{3}\right) .
\end{aligned}
$$

Then $E\left(P_{1}\right)=0$ and $P_{1}+P_{2}+P_{3}=0$ yield

$$
a+b+c=0, \quad 3 b+2 f=0, \quad c+3 d+2 f=0, \quad d+e=0 .
$$

Hence we obtain

$$
a=-e-\frac{4}{3} f, \quad b=-\frac{2}{3} f, \quad c=e-\frac{2}{3} f, \quad d=-e .
$$

Therefore st $(5,4)=2$ and we can compute two pairs of polynomials that provide explicit basis polynomials for each standard representation in $\operatorname{Ker}(E) \cap A(5)_{4}$ :

(i) if we take $e=-1, f=0$, then we have

$$
\begin{aligned}
& P_{1}=\left(x_{1}^{4}-x_{2}^{4}\right)-\left(x_{1}^{3} x_{3}-x_{2}^{3} x_{3}\right)+\left(x_{1}^{2} x_{3}^{2}-x_{2}^{2} x_{3}^{2}\right)-\left(x_{1} x_{3}^{3}-x_{2} x_{3}^{3}\right), \\
& P_{2}=\left(x_{2}^{4}-x_{3}^{4}\right)-\left(x_{1} x_{2}^{3}-x_{1} x_{3}^{3}\right)+\left(x_{1}^{2} x_{2}^{2}-x_{1}^{2} x_{3}^{2}\right)-\left(x_{1}^{3} x_{2}-x_{1}^{3} x_{3}\right) .
\end{aligned}
$$

(ii) if we take $e=0, f=-3$, then we have

$$
\begin{aligned}
& P_{1}=4\left(x_{1}^{4}-x_{2}^{4}\right)+2\left(x_{1}^{3} x_{2}-x_{1} x_{2}^{3}\right)+2\left(x_{1}^{3} x_{3}-x_{2}^{3} x_{3}\right)-3\left(x_{1}^{2} x_{2} x_{3}-x_{1} x_{2}^{2} x_{3}\right), \\
& P_{2}=4\left(x_{2}^{4}-x_{3}^{4}\right)+2\left(x_{2}^{3} x_{3}-x_{2} x_{3}^{3}\right)+2\left(x_{1} x_{2}^{3}-x_{1} x_{3}^{3}\right)-3\left(x_{1} x_{2}^{2} x_{3}-x_{1} x_{2} x_{3}^{2}\right) .
\end{aligned}
$$

\section{Multiplicities of irReducible Submodules: ReCursive formulas}

In this section, we will give recursive formulas for the multiplicities of the irreducible submodules in $\operatorname{Ker}(E) \cap A(d)_{j}$ for all $d \geq 3, j \geq 0$.

Recall that we denote by $\mathscr{P}_{k, l}(j)$ (resp. $\left.\mathscr{P}_{k, l}^{+}(j)\right)$ be the set of rectangular partitions (resp. strict partitions) of $j$ of size $k \times l$, and let $p_{k, l}(j)$ (resp. $p_{k, l}^{+}(j)$ ) be the number of elements in $\mathscr{P}_{k, l}(j)$ (resp. $\mathscr{P}_{k, l}^{+}(j)$ ). We say that a partition $\lambda=\left(\lambda_{1} \geq \lambda_{2} \geq \ldots \geq \lambda_{r} \geq 0\right)$ is 2 -strict if $\lambda_{j}-\lambda_{j+1} \geq 2$ for all $1 \leq j \leq r$. We will use the notation $\mathscr{P}_{k, l}^{+}(j)$ for the set of 2 -strict rectangular partitions of size $k \times l$ and $p_{k, l}^{++}(j)$ the number of elements in $\mathscr{P}_{k, l}^{++}(j)$.

Let $\Delta=(k-1, k-2, \ldots, 1,0)$ be a rectangular partition of $\frac{k(k-1)}{2}$ of size $k \times(k-1)$. Then it is straightforward to see that there is a bijection

$$
\mathscr{P}^{+}(j) \stackrel{\sim}{\longrightarrow} \mathscr{P}_{k, l-k+1}\left(j-\frac{k(k-1)}{2}\right) \quad \text { given by } \lambda \longmapsto \lambda-\Delta .
$$

Similarly, we have

$$
\mathscr{P}_{k, l}^{++}(j) \stackrel{\sim}{\longrightarrow} \mathscr{P}_{k, l-k+1}^{+}\left(j-\frac{k(k-1)}{2}\right) .
$$

In particular, when $k=3, l=d-1$, we have

$$
\mathscr{P}_{3, d-1}^{+}(j) \stackrel{\sim}{\longrightarrow} \mathscr{P}_{3, d-3}(j-3), \quad \mathscr{P}_{3, d-1}^{++}(j) \stackrel{\sim}{\longrightarrow} \mathscr{P}_{3, d-3}^{+}(j-3) .
$$

Therefore, we obtain the following recursive relations. 
Proposition 4.1. For all $d \geq 3, j \geq 0$, we have

$$
\operatorname{sign}(d, j)=\operatorname{triv}(d-2, j-3) .
$$

Proof. By Theorem 3.7 and (4.1), we have

$$
\operatorname{sign}(d, j)=p_{3, d-1}^{+}(j)-p_{3, d-1}^{+}(j-1)=p_{3, d-3}(j-3)-p_{3, d-3}(j-4)=\operatorname{triv}(d-2, j-3) .
$$

In the next proposition, we will prove the opposite direction of recursive relations.

Proposition 4.2. For all $d \geq 3, j \geq 0$, we have

$$
\operatorname{triv}(d, j)= \begin{cases}\operatorname{sign}(d-2, j-3)+1 & \text { if } j \leq d-1, \\ \operatorname{sign}(d-2, j-3) & \text { if } j \geq d .\end{cases}
$$

Proof. Note that

$$
p_{3, d-1}^{++}(j)-p_{3, d-1}^{++}(j-1)=p_{3, d-3}^{+}(j-3)-p_{3, d-3}^{+}(j-4)=\operatorname{sign}(d-2, j-3) .
$$

Hence our claim is equivalent to the relations

$$
p_{3, d-1}(j)-p_{3, d-1}(j-1)= \begin{cases}p_{3, d-1}^{++}(j)-p_{3, d-1}^{++}(j-1)+1 & \text { if } j \leq d-1, \\ p_{3, d-1}^{++}(j)-p_{3, d-1}^{++}(j-1) & \text { if } j \geq d .\end{cases}
$$

We will prove our claim in 4 steps.

Step 1: For all $d \geq 3, j \geq 0$, we have

$$
\begin{aligned}
& \left(p_{3, d-1}(j)-p_{3, d-1}(j-1)\right)-\left(p_{3, d-1}^{++}(j)-p_{3, d-1}^{++}(j-1)\right) \\
& =\#\left\{\lambda=\left(\lambda_{1}, \lambda_{2}, \lambda_{3} \in \mathscr{P}_{3, d-1}(j) \mid \lambda_{1}=\lambda_{2}\right\}\right. \\
& \quad-\#\left\{\lambda=\left(\lambda_{1}, \lambda_{2}, \lambda_{3} \in \mathscr{P}_{3, d-1}(j) \mid \lambda_{1}=\lambda_{2}+2, \lambda \text { is 2-struct }\right\}\right. \\
& \quad-\#\left\{\mu=\left(\mu_{1}, \mu_{2}, \mu_{3}\right) \in \mathscr{P}_{3, d-1}(j-1) \mid \mu_{1}=d-1, \mu \text { is not 2-strict }\right\} .
\end{aligned}
$$

Roughly speaking, our main idea is to use the following map

$$
\mathscr{P}_{3, d-1}(j) \ni \lambda=\left(\lambda_{1}, \lambda_{2}, \lambda_{3}\right) \longmapsto \mu=\left(\lambda_{1}-1, \lambda_{2}, \lambda_{3}\right) \in \mathscr{P}_{3, d-1}(j-1)
$$

whenever it makes sense and add the missing partitions. For simplicity, we will just use $\lambda$ and $\mu$ for the partitions in $\mathscr{P}_{3, d-1}(j)$ and $\mathscr{P}_{3, d-1}(j-1)$, respectively.

We first observe

$$
\begin{aligned}
& p_{3, d-1}(j)-p_{3, d-1}(j-1) \\
& =\#\left\{\lambda \mid \lambda_{1}=\lambda_{2}\right\}+\#\left\{\lambda \mid \lambda_{1}=\lambda_{2}+1\right\}-\#\left\{\mu \mid \mu_{1}=\mu_{2}\right\} \\
& \quad+\#\left\{\lambda \mid \lambda_{1}=\lambda_{2}+2\right\}-\#\left\{\mu \mid \mu_{1}=\mu_{2}+1\right\} \\
& \quad+\#\left\{\lambda \mid \lambda_{1} \geq \lambda_{2}+3, \lambda \text { is } 2 \text {-strict }\right\}+\#\left(\left\{\lambda \mid \lambda_{1} \geq \lambda_{2}+3, \lambda \text { is not 2-strict }\right\}\right. \\
& \quad-\#\{\mu \mid \mu \text { is } 2 \text {-strict }\}-\#\left(\left\{\mu \mid \mu_{1} \geq \mu_{2}+2, \mu \text { is not } 2 \text {-strict }\right\}\right. \\
& =\#\left\{\lambda \mid \lambda_{1}=\lambda_{2}\right\}-\#\left\{\mu \mid \mu_{1}=d-1\right\} .
\end{aligned}
$$

On the other hand, we have

$$
\begin{aligned}
& p_{3, d-1}^{++}(j)-p_{3, d-1}^{++}(j-1) \\
& =\#\left\{\lambda \mid \lambda_{1} \geq \lambda_{2}+3, \lambda \text { is 2-strict }\right\}-\#\{\mu \mid \mu \text { is 2-strict }\}+\#\left\{\lambda \mid \lambda_{1}=\lambda_{2}+2, \lambda \text { is 2-strict }\right\} \\
& =\#\left\{\lambda \mid \lambda_{1}=\lambda_{2}+2, \lambda \text { is 2-strict }\right\}-\#\left\{\mu \mid \mu_{1}=d-1, \mu \text { is 2-strict }\right\} .
\end{aligned}
$$


Hence we obtain

$$
\begin{aligned}
& \left(p_{3, d-1}(j)-p_{3, d-1}(j-1)\right)-\left(p_{3, d-1}^{++}(j)-p_{3, d-1}^{++}(j-1)\right) \\
& =\#\left\{\lambda \mid \lambda_{1}=\lambda_{2}\right\}-\#\left\{\lambda \mid \lambda_{1}=\lambda_{2}+2, \lambda \text { is 2-struct }\right\}-\#\left\{\mu \mid \mu_{1}=d-1, \mu \text { is not 2-strict }\right\} .
\end{aligned}
$$

Step 2: If $\lambda=(a, a, b) \in \mathscr{P}_{3, d-1}(j)$ for $d \geq 3, j \geq 0$, then $a+1 \leq d-1$.

To prove this, since $j=2 a+b$. we have only to check our claim when $j$ and $d$ are maximal.

If $d=3$, then $j=3 a=1$, which verifies our claim. If $d$ is odd $\geq 5$, then we have

$$
j=\frac{3(d-1)}{2} \text { and } a \leq \frac{3(d-1)}{4} .
$$

Hence we obtain

$$
(d-1)-(a+1) \geq(d-1)-\left(\frac{3(d-1)}{4}+1\right)=\frac{d-5}{4} \geq 0 .
$$

If $d$ is even, then $d \geq 4$ and

$$
j=\left\lfloor\frac{3(d-1)}{2}\right\rfloor=\frac{3 d-4}{2}, \quad a \leq \frac{3 d-4}{4} .
$$

Hence we obtain

$$
(d-1)-(a+1) \geq(d-1)-\left(\frac{3 d-4}{4}+1\right)=\frac{d-4}{4} \geq 0,
$$

which proves our claim.

Step 3: $\#\left\{\lambda \in \mathscr{P}_{3, d-1}(j) \mid \lambda_{1}=\lambda_{2}\right\}=\#\left\{\lambda \in \mathscr{P}_{3, d-1}(j) \mid \lambda_{1}=\lambda_{2}+2, \lambda\right.$ is 2-strict $\}+1$.

Set

$$
\begin{aligned}
& L=\left\{\lambda \in \mathscr{P}_{3, d-1}(j) \mid \lambda_{1}=\lambda_{2}\right\}, \quad L^{+}=\left\{\lambda \in \mathscr{P}_{3, d-1}(j) \mid \lambda=\lambda_{2} \geq \lambda_{3}+3\right\}, \\
& R=\left\{\lambda \in \mathscr{P}_{3, d-1}(j) \mid \lambda_{1}=\lambda_{2}+2, \lambda \text { is 2-strict }\right\} .
\end{aligned}
$$

Then it is easy to see that there is a bijection

$$
L^{+} \stackrel{\sim}{\longrightarrow} R \text { given by } \lambda=(a, a, b) \longmapsto \lambda^{\prime}=(a+1, a-1, b),
$$

which implies $L \backslash L^{+}=\left\{\lambda=(a, a, b) \mid \lambda_{1}=\lambda_{2} \leq \lambda_{3}+2\right\}$.

Therefore, we have the following three cases:

(i) if $j \equiv 1(\bmod 3)$, then $a=b+2$ and $L \backslash L^{+}=\{(a, a, a-2)\}$,

(ii) if $j \equiv 2(\bmod 3)$, then $a=b+1$ and $L \backslash L^{+}=\{(a, a, a-1)\}$,

(iii) if $j \equiv 0 \quad(\bmod 3)$, then $a=b$ and $L \backslash L^{+}=\{(a, a, a)\}$.

Hence $\#\left(L \backslash L^{+}\right)=1$.

\section{Step 4:}

(a) If $j \leq d-1$, then $\left\{\mu \in \mathscr{P}_{3, d-1}(j-1) \mid \mu_{1}=d-1, \mu\right.$ is not 2 -strict $\}=\emptyset$.

(b) If $j \geq d$, then $\#\left(\left\{\mu \in \mathscr{P}_{3, d-1}(j-1) \mid \mu_{1}=d-1, \mu\right.\right.$ is not 2-strict $\left.\}\right)=1$.

The statement (a) is clear because $\mu=(d-1, a, b)$ cannot be a partition of $j-1$.

Suppose $j \geq d$. We begin with $\mu_{0}=(d-1, j-d, 0)$. If $\mu_{0}$ is not 2-strict, then $j-d=1$ and we stop there. Otherwise, we continue to obtain $\mu_{k}=(d-1, j-d-k, k)$, where $k$ is the largest non-negative integer such that $\mu_{k}$ is 2 -strict. Then $j-d-k \geq k+2$ and $\mu_{k+1}=(d-1, j-d-k-1, k+1)$, which is not 2-strict; i.e., $j-d-k-1 \leq 1$. Then $\mu_{k+2}=(d-1, j-d-k-2, k+2)$ is not even a partition and our assertion is proved. 
Combining (Step 1) - (Step 4), we obtain (4.6).

Corollary 4.3. For all $d \geq 3, j \geq 0$, we have

$$
\operatorname{st}(d, j)= \begin{cases}\operatorname{st}(d-2, j-3)+1 & \text { if } j \leq d-1, \\ \operatorname{st}(d-2, j-3) & \text { if } j \geq d .\end{cases}
$$

Proof. Note that

$$
\begin{aligned}
& 2(\operatorname{st}(d, j)-\operatorname{st}(d-2, j-3))+(\operatorname{triv}(d, j)-\operatorname{triv}(d-2, j-3))+(\operatorname{sign}(d, j)-\operatorname{sign}(d-2, j-3)) \\
& =\operatorname{mult}(d, j)-\operatorname{mult}(d-2, j-3) .
\end{aligned}
$$

Let $L$ (resp. $R$ ) denote the left-hand side (resp. right-hand side) of (4.8).

If $j \leq d-1$, then

$$
L=2(\operatorname{st}(d, j)-\operatorname{st}(d-2, j-3))+1, \quad R=(j+1)-(j-2)=3 .
$$

Hence we get

$$
\operatorname{st}(d, j)-\operatorname{st}(d-2, j-3)=1 .
$$

If $j \geq d+1$, then

$$
L=2(\operatorname{st}(d, j)-\operatorname{st}(d-2, j-3)), \quad R=(3 d-2-2 j)-(3(d-2)-2-2(j-3))=0,
$$

which yields

$$
\operatorname{st}(d, j)-\operatorname{st}(d-2, j-3)=0 .
$$

If $j=d$, then

$$
L=2(\operatorname{st}(d, d)-\operatorname{st}(d-2, d-3)), \quad R=(3 d-2-2 d)-(d-2)=0,
$$

which completes the proof.

Example 4.4. In this example, we illustrate how to compute multiplicities irreducible components using the resursive formulas (4.4), (4.5) and (4.7).

(a) Suppose that $d=9$.

(i) If $j=8$, then we have

$$
\begin{aligned}
& \operatorname{triv}(9,8)=\operatorname{sign}(7,5)+1=\operatorname{triv}(5,2)+1=\operatorname{sign}(3,-2)+2=2, \\
& \operatorname{sign}(9,8)=\operatorname{triv}(7,5)=\operatorname{sign}(5,2)+1=\operatorname{triv}(3,-2)+1=1, \\
& \operatorname{st}(9,8)=\operatorname{st}(7,5)+1=\operatorname{st}(5,2)+2=1+2=3 .
\end{aligned}
$$

(ii) If $j=12$, then we have

$$
\begin{aligned}
& \operatorname{triv}(9,12)=\operatorname{sign}(7,9)=\operatorname{triv}(5,6)=\operatorname{sign}(3,3)=1, \\
& \operatorname{sign}(9,12)=\operatorname{triv}(7,9)=\operatorname{sign}(5,6)=\operatorname{triv}(3,3)=0, \\
& \operatorname{st}(9,12)=\operatorname{st}(7,9)=\operatorname{st}(5,6)=\mathrm{t}(3,3)=0 .
\end{aligned}
$$

(iii) If $j=6$, then we have

$$
\begin{aligned}
& \operatorname{triv}(9,6)=\operatorname{sign}(7,3)+1=\operatorname{triv}(5,0)+1=1=1=2, \\
& \operatorname{sign}(9,6)=\operatorname{triv}(7,3)=\operatorname{sign}(5,0)+1=1, \\
& \operatorname{st}(9,6)=\operatorname{st}(7,3)+1=\operatorname{st}(5,0)+2=2 .
\end{aligned}
$$

(b) Suppose that $d=10$. 
(i) If $j=9$, then we have

$$
\begin{aligned}
& \operatorname{triv}(10,9)=\operatorname{sign}(8,6)+1=\operatorname{triv}(6,3)+1=\operatorname{sign}(4,0)+2=2, \\
& \operatorname{sign}(10,9)=\operatorname{triv}(8,6)=\operatorname{sign}(6,3)+1=\operatorname{triv}(4,0)+1=1+1=2, \\
& \operatorname{st}(10,9)=\operatorname{st}(8,6)+1=\operatorname{st}(6,3)+2=\operatorname{st}(4,0)+3=3 .
\end{aligned}
$$

(ii) If $j=12$, then we have

$$
\begin{aligned}
& \operatorname{triv}(10,12)=\operatorname{sign}(8,9)=\operatorname{triv}(6,6)=\operatorname{sign}(4,3)=1, \\
& \operatorname{sign}(10,12)=\operatorname{triv}(8,9)=\operatorname{sign}(6,6)=\operatorname{triv}(4,3)=1, \\
& \operatorname{st}(10,12)=\operatorname{st}(8,9)=\operatorname{st}(6,6)=\operatorname{st}(4,3)=1 .
\end{aligned}
$$

(iii) If $j=6$, then we have

$$
\begin{aligned}
& \operatorname{triv}(10,6)=\operatorname{sign}(8,3)+1=\operatorname{triv}(5,0)+1=1+1=2, \\
& \operatorname{sign}(10,6)=\operatorname{triv}(8,3)=\operatorname{sign}(6,0)+1=1, \\
& \operatorname{st}(10,6)=\operatorname{st}(8,3)+1=\operatorname{st}(6,0)+2=2 .
\end{aligned}
$$

\section{Multiplicities of iRREduCiBle Submodules: ClOSED FORM FORMUlas}

In this section, using the recursive formulas in Proposition 4.1, Proposition 4.2 and Corollary 4.3, we derive explicit closed form formulas for the multiplicities of irreducible submodules in $\operatorname{Ker}(E) \cap A(d)_{j}$ for all $d \geq 3$, $j \geq 0$.

We first take care of the case when $j=d-1$, which will play the role of corner-stone of our closed form formulas.

\section{Lemma 5.1.}

(a) If $d$ is odd $\geq 3$, then we have

$$
\operatorname{triv}(d, d-1)=\left\lfloor\frac{d-1}{6}\right\rfloor+1, \quad \operatorname{sign}(d, d-1)=\left\lfloor\frac{d-1}{6}\right\rfloor .
$$

(b) If $d$ is even $\geq 4$, then we have

$$
\operatorname{triv}(d, d-1)=\operatorname{sign}(d, d-1)=\left\lfloor\frac{d+2}{6}\right\rfloor .
$$

Proof. Since $d-1 \leq d$, by the recursive formulas (4.4) and (4.5), we have

$$
\begin{aligned}
& \operatorname{triv}(d, d-1)=\operatorname{sign}(d-2, d-4)+1 \\
& \quad=\operatorname{triv}(d-4, d-7)+1=\operatorname{sign}(d-6, d-10)+2 \\
& \quad=\cdots=\operatorname{sign}(d-4 l+2, d-6 l+2)+l=\operatorname{triv}(d-4 l, d-6 l-1)+l, \\
& \operatorname{sign}(d, d-1)=\operatorname{triv}(d-2, d-4) \\
& \quad=\operatorname{sign}(d-4, d-7)+1=\operatorname{triv}(d-6, d-10)+1 \\
& \quad=\cdots=\operatorname{triv}(d-4 l+2, d-6 l+2)+l-1=\operatorname{sign}(d-4 l, d-6 l-1)+l .
\end{aligned}
$$

(a) Suppose $d$ is odd and $d \geq 3$. 
If $d=6 l+1$, then

$$
\begin{aligned}
& \operatorname{triv}(d, d-1)=\operatorname{triv}(2 l+1,0)+l=l+1=\frac{d-1}{6}+1=\left\lfloor\frac{d-1}{6}\right\rfloor+1, \\
& \operatorname{sign}(d, d-1)=\operatorname{sign}(2 l+1,0)+l=l=\left\lfloor\frac{d-1}{6}\right\rfloor .
\end{aligned}
$$

If $d=6 l+3$, then

$$
\begin{aligned}
& \operatorname{triv}(d, d-1)=\operatorname{triv}(2 l+3,2)+l=l+1=\frac{d-3}{6}+1=\left\lfloor\frac{d-3}{6}\right\rfloor+1 \\
& \quad=\left\lfloor\frac{d-3}{6}+\frac{1}{3}\right\rfloor+1=\left\lfloor\frac{d-1}{6}\right\rfloor+1, \\
& \operatorname{sign}(d, d-1)=\operatorname{sign}(2 l+3,2)+l=l=\left\lfloor\frac{d-3}{6}\right\rfloor=\left\lfloor\frac{d-1}{6}\right\rfloor .
\end{aligned}
$$

If $d=6 l+5$, then

$$
\begin{aligned}
& \operatorname{triv}(d, d-1)=\operatorname{triv}(2 l+5,4)+l=\operatorname{sign}(2 l+3,1)+l+1 \\
& \quad=l+1=\left\lfloor\frac{d-5}{6}\right\rfloor+1=\left\lfloor\frac{d-1}{6}\right\rfloor+1, \\
& \operatorname{sign}(d, d-1)=\operatorname{sign}(2 l+5,4)+l=\operatorname{triv}(2 l+3,1)+l=l=\left\lfloor\frac{d-1}{6}\right\rfloor .
\end{aligned}
$$

(b) Suppose $d$ is even and $d \geq 4$.

If $d=6 l$, then

If $d=6 l+2$, then

$$
\begin{aligned}
& \operatorname{triv}(d, d-1)=\operatorname{triv}(2 l,-1)+l=l=\frac{d}{6}=\left\lfloor\frac{d}{6}\right\rfloor=\left\lfloor\frac{d+2}{6}\right\rfloor, \\
& \operatorname{sign}(d, d-1)=\operatorname{sign}(2 l,-1)+l=l=\left\lfloor\frac{d+2}{6}\right\rfloor .
\end{aligned}
$$

$$
\begin{aligned}
& \operatorname{triv}(d, d-1)=\operatorname{triv}(2 l+2,1)+l=l=\frac{d-2}{6}=\left\lfloor\frac{d-2}{6}\right\rfloor=\left\lfloor\frac{d+2}{6}\right\rfloor, \\
& \operatorname{sign}(d, d-1)=\operatorname{sign}(2 l+2,1)+l=l=\left\lfloor\frac{d+2}{6}\right\rfloor .
\end{aligned}
$$

If $d=6 l+4$, then

$$
\begin{aligned}
& \operatorname{triv}(d, d-1)=\operatorname{triv}(2 l+4,3)+l=\operatorname{sign}(2 l+2,0)+l=\frac{d-2}{6}=\left\lfloor\frac{d-2}{6}\right\rfloor=\left\lfloor\frac{d+2}{6}\right\rfloor, \\
& \operatorname{sign}(d, d-1)=\operatorname{sign}(2 l+4,3)+l=l+1=\left\lfloor\frac{d-4}{6}+1\right\rfloor=\left\lfloor\frac{d+2}{6}\right\rfloor .
\end{aligned}
$$

Example 5.2. Lemma 5.1 implies

$$
\begin{aligned}
& \operatorname{triv}(9,8)=\left\lfloor\frac{9-1}{6}\right\rfloor+1=2, \\
& \operatorname{sign}(9,8)=\left\lfloor\frac{9-1}{6}\right\rfloor=1 \\
& \operatorname{triv}(10,9)=\operatorname{sign}(10,9)=\left\lfloor\frac{10+2}{6}\right\rfloor=2 .
\end{aligned}
$$


Now using Lemma 5.1, we will derive the general multiplicity formulas for $\operatorname{triv}(d, j)$ and $\operatorname{sign}(d, j)$. Note that, thanks to recursive formulas (4.4) and (4.5), we have the interlocking relations

$$
\begin{aligned}
& \operatorname{triv}(d, j)=\operatorname{sign}(d+2, j+3) \text { for all } d \geq 3, j \geq 0, \\
& \operatorname{sign}(d, j)= \begin{cases}\operatorname{triv}(d+2, j+3)-1 & \text { if } j \leq d-2, \\
\operatorname{triv}(d+2, j+3) & \text { if } j \geq d-1 .\end{cases}
\end{aligned}
$$

Suppose $j \geq d$. Then we have

$$
\begin{aligned}
& \operatorname{triv}(d, j)=\operatorname{sign}(d-2, j-3) \\
& \quad=\operatorname{triv}(d-4, j-6)=\operatorname{sign}(d-6, j-9) \\
& \quad=\cdots=\operatorname{sign}(d-4 l+2, j-6 l+3)=\operatorname{triv}(d-4 l, j-6 l)=\cdots, \\
& \operatorname{sign}(d, j)=\operatorname{triv}(d-2, j-3) \\
& \quad=\operatorname{sign}(d-4, j-6)=\operatorname{triv}(d-6, j-9) \\
& \quad=\cdots=\operatorname{triv}(d-4 l+2, d-6 l+3)=\operatorname{sign}(d-4 l, j-6 l)=\cdots .
\end{aligned}
$$

We continue this process until we reach the point where

$$
d-4 l+1=j-6 l+3 \text { or } d-4 l-1=j-6 l .
$$

If $d-4 l+1=j-6 l+3$, then $j-d=2(l-1)$ is even and $l=\frac{j-d+2}{2}$.

If $d-4 l-1=j-6 l$, then $j-d=2 l-1$ is odd and $l=\frac{j-d+1}{2}$.

Now we will compute $\operatorname{triv}(d, j)$ and $\operatorname{sign}(d, j)$ when $j \geq d$.

Case 1: If $d$ is odd and $j-d$ is even, then we have

$$
\begin{aligned}
& \operatorname{triv}(d, j)=\operatorname{sign}(d-4 l+2, j-6 l+3)=\left\lfloor\frac{d-4 l+1}{6}\right\rfloor \\
& \quad=\left\lfloor\frac{d-2(j-d+2)+1}{6}\right\rfloor=\left\lfloor\frac{3 d-2 j-3}{6}\right\rfloor, \\
& \operatorname{sign}(d, j)=\operatorname{triv}(d,-4 l+2, j-6 l+3)=\left\lfloor\frac{d-4 l+1}{6}\right\rfloor+1 \\
& =\left\lfloor\frac{3 d-2 j-3}{6}\right\rfloor+1 .
\end{aligned}
$$

Case 2: If $d$ is odd and $j-d$ is odd, then

$$
\begin{aligned}
& \operatorname{triv}(d, j)=\operatorname{triv}(d-4 l, j-6 l)=\left\lfloor\frac{d-4 l-1}{6}\right\rfloor+1 \\
& \quad=\left\lfloor\frac{d-2(j-d+1)-1}{6}\right\rfloor+1=\left\lfloor\frac{3 d-2 j-3}{6}\right\rfloor+1 \\
& \operatorname{sign}(d, j)=\operatorname{sign}(d,-4 l, j-6 l)=\left\lfloor\frac{d-4 l-1}{6}\right\rfloor \\
& \quad=\left\lfloor\frac{3 d-2 j-3}{6}\right\rfloor .
\end{aligned}
$$


Case 3: If $d$ is even and $j-d$ is even, then

$$
\begin{aligned}
& \operatorname{triv}(d, j)=\operatorname{sign}(d, j)=\operatorname{sign}(d-4 l+2, j-6 l+3) \\
& \quad=\left\lfloor\frac{d-4 l+4}{6}\right\rfloor=\left\lfloor\frac{d-2(j-d+2)+4}{6}\right\rfloor=\left\lfloor\frac{3 d-2 j}{6}\right\rfloor .
\end{aligned}
$$

Case 4: If $d$ is even and $j-d$ is odd, then

$$
\begin{aligned}
& \operatorname{triv}(d, j)=\operatorname{sign}(d, j)=\operatorname{sign}(d-4 l, j-6 l) \\
& \quad=\left\lfloor\frac{d-4 l+2}{6}\right\rfloor=\left\lfloor\frac{d-2(j-d+1)+2}{6}\right\rfloor=\left\lfloor\frac{3 d-2 j}{6}\right\rfloor .
\end{aligned}
$$

To summarize, we obtain the following closed form formulas.

Theorem 5.3. Suppose that $j \geq d$.

(a) If $d$ is odd and $j-d$ is even, then we have

$$
\operatorname{triv}(d, j)=\left\lfloor\frac{3 d-2 j-3}{6}\right\rfloor, \quad \operatorname{sign}(d, j)=\left\lfloor\frac{3 d-2 j-3}{6}\right\rfloor+1 .
$$

(b) If $d$ is odd and $j-d$ is odd, then we have

$$
\operatorname{triv}(d, j)=\left\lfloor\frac{3 d-2 j-3}{6}\right\rfloor+1, \quad \operatorname{sign}(d, j)=\left\lfloor\frac{3 d-2 j-3}{6}\right\rfloor .
$$

(c) If $d$ is even, then we have

$$
\operatorname{triv}(d, j)=\operatorname{sign}(d, j)=\left\lfloor\frac{3 d-2 j}{6}\right\rfloor
$$

Example 5.4. By Theorem 5.3, we have

$$
\begin{aligned}
& \operatorname{triv}(9,12)=\left\lfloor\frac{27-24-3}{6}\right\rfloor+1=1, \quad \operatorname{sign}(9,12)=\left\lfloor\frac{27-24-3}{6}\right\rfloor=0, \\
& \operatorname{triv}(10,12)=\operatorname{sign}(10,12)=\left\lfloor\frac{30-24}{6}\right\rfloor=1 .
\end{aligned}
$$

Next, we will consider the case $j \leq d-2$. By the recursive relations (5.1), we have

$$
\begin{aligned}
& \operatorname{triv}(d, j)=\operatorname{sign}(d+2, j+3) \\
& \quad=\operatorname{triv}(d+4, j+6)-1=\operatorname{sign}(d+6, j+9)-1 \\
& \quad=\cdots=\operatorname{sign}(d+4-+2, j+6 l-3)-l+1=\operatorname{triv}(d+4 l, j+6 l)-l=\cdots, \\
& \operatorname{sign}(d, j)=\operatorname{triv}(d+2, j+3-1 \\
& \quad=\operatorname{sign}(d+4, j+6)-1=\operatorname{triv}(d+6, j+9)-2 \\
& \quad=\cdots=\operatorname{triv}(d+4 l-2, d+6 l-3)-l=\operatorname{sign}(d+4 l, j+6 l)-l=\cdots .
\end{aligned}
$$

If $d+4 l-3=j+6 l-3$, then $d-j=2 l$ is even and $l=\frac{d-j}{2}$.

If $d+4 l-1=j+6 l$, then $d-j=2 l+1$ is odd and $l=\frac{d-j-1}{2}$.

We will compute $\operatorname{triv}(d, j)$ and $\operatorname{sign}(d, j)$ for $j \leq d-2$. 
Case 1: If $d$ is odd and $d-j$ is even, then we have

$$
\begin{aligned}
& \operatorname{triv}(d, j)=\operatorname{sign}(d+4 l-2, j+6 l-3)-l+1=\left\lfloor\frac{d+4 l-3}{6}\right\rfloor-l+1 \\
& =\left\lfloor\frac{d+2(d-j)+3}{6}\right\rfloor-\frac{d-j}{2}+1=\left\lfloor\frac{3 d-2 j-3}{6}\right\rfloor-\left(\frac{d-j-2}{2}\right), \\
& \operatorname{sign}(d, j)=\operatorname{triv}(d,+4 l-2, j+6 l-3)-l=\left(\left\lfloor\frac{d+4 l-3}{6}\right\rfloor+1\right)-\frac{d-j}{2} \\
& =\left\lfloor\frac{3 d-2 j-3}{6}\right\rfloor-\left(\frac{d-j-2}{2}\right) .
\end{aligned}
$$

Case 2: If $d$ is odd and $d-j$ is odd, then we have

$$
\begin{aligned}
& \operatorname{triv}(d, j)=\operatorname{triv}(d+4 l, j+6 l)-l=\left\lfloor\frac{d+4 l-1}{6}\right\rfloor+1-l \\
& =\left\lfloor\frac{d+2(d-j+1)-1}{6}\right\rfloor+1-\frac{d-j+1}{2}=\left\lfloor\frac{3 d-2 j-3}{6}\right\rfloor-\left(\frac{d-j-3}{2}\right), \\
& \operatorname{sign}(d, j)=\operatorname{triv}(d,+4 l, j+6 l)-l=\left(\left\lfloor\frac{d+4 l-1}{6}\right\rfloor\right)-\frac{d-j-1}{2} \\
& =\left\lfloor\frac{3 d-2 j-3}{6}\right\rfloor-\left(\frac{d-j-1}{2}\right) .
\end{aligned}
$$

Case 3: If $d$ is even and $d-j$ is even, then we have

$$
\begin{aligned}
& \operatorname{triv}(d, j)=\operatorname{sign}(d+4 l-2, j+6 l-3)-l+1=\left\lfloor\frac{d+4 l}{6}\right\rfloor-l+1 \\
& =\left\lfloor\frac{d+2(d-j)}{6}\right\rfloor-\frac{d-j}{2}+1=\left\lfloor\frac{3 d-2 j}{6}\right\rfloor-\left(\frac{d-j-2}{2}\right), \\
& \operatorname{sign}(d, j)=\operatorname{triv}(d,+4 l-2, j+6 l-3)-l=\left\lfloor\frac{d+4 l}{6}\right\rfloor-\frac{d-j}{2} \\
& =\left\lfloor\frac{3 d-2 j}{6}\right\rfloor-\left(\frac{d-j}{2}\right) .
\end{aligned}
$$

Case 4: If $d$ is even and $d-j$ is odd, then we have

$$
\begin{aligned}
& \operatorname{triv}(d, j)=\operatorname{triv}(d+4 l, j+6 l)-l=\left\lfloor\frac{d+4 l+2}{6}\right\rfloor-l \\
& =\left\lfloor\frac{d+2(d-j-1)+2}{6}\right\rfloor-\frac{d-j+1}{2}=\left\lfloor\frac{3 d-2 j}{6}\right\rfloor-\left(\frac{d-j-1}{2}\right), \\
& \operatorname{sign}(d, j)=\operatorname{sign}(d,+4 l, j+6 l)-l=\left\lfloor\frac{d+4 l+2}{6}\right\rfloor-\frac{d-j-1}{2} \\
& =\left\lfloor\frac{3 d-2 j}{6}\right\rfloor-\left(\frac{d-j-1}{2}\right) .
\end{aligned}
$$

Moreover, note that the case $j=d-1$ is included in (Case 2) and (Case 4).

Therefore we obtain:

Theorem 5.5. Suppose $j \leq d-1$.

(a) If $d$ is odd and $d-j$ is even, then we have

$$
\operatorname{triv}(d, j)=\operatorname{sign}(d, j)=\left\lfloor\frac{3 d-2 j-3}{6}\right\rfloor-\left(\frac{d-j-2}{2}\right) .
$$


(b) If $d$ is odd and $d-j$ is odd, then we have

$$
\operatorname{triv}(d, j)=\left\lfloor\frac{3 d-2 j-3}{6}\right\rfloor-\left(\frac{d-j-3}{2}\right), \operatorname{sign}(d, j)=\left\lfloor\frac{3 d-2 j-3}{6}\right\rfloor-\left(\frac{d-j-1}{2}\right) .
$$

(c) If $d$ is even and $d-j$ is even, then we have

$$
\operatorname{triv}(d, j)=\left\lfloor\frac{3 d-2 j}{6}\right\rfloor-\left(\frac{d-j-2}{2}\right), \operatorname{sign}(d, j)=\left\lfloor\frac{3 d-2 j}{6}\right\rfloor-\left(\frac{d-j}{2}\right) .
$$

(d) if $d$ is even and $d-j$ is odd, then we have

$$
\operatorname{triv}(d, j)=\operatorname{sign}(d, j)=\left\lfloor\frac{3 d-2 j}{6}\right\rfloor-\left(\frac{d-j-1}{2}\right) .
$$

Example 5.6. By Theorem 5.5, we have

$$
\begin{aligned}
& \operatorname{triv}(9,6)=\left\lfloor\frac{27-12-3}{6}\right\rfloor-\left(\frac{9-6-3}{2}\right)=2=0=2, \\
& \operatorname{sign}(9,6)=\left\lfloor\frac{27-12-3}{6}\right\rfloor-\left(\frac{9-6-1}{2}\right)=2-1=1, \\
& \operatorname{triv}(10,6)=\left\lfloor\frac{30-12}{6}\right\rfloor-\left(\frac{10-6-2}{2}\right)=3-1=2, \\
& \operatorname{sign}(10,6)=\left\lfloor\frac{30-12}{6}\right\rfloor-\left(\frac{10-6}{2}\right)=3-2=1 .
\end{aligned}
$$

We will now move on to standard representations. Assume first that $j=d-1$. By the resursive formula (4.7), we have

$$
\begin{aligned}
& \operatorname{st}(d, d-1)=\operatorname{st}(d-2, d-4)+1=\operatorname{st}(d-6, d-7)+2 \\
& \quad=\cdots=\operatorname{st}(d-2 l, d-3 l-1)+l .
\end{aligned}
$$

If $d=3 l$, then

If $d=3 l+1$, then

$$
\operatorname{st}(d, d-1)=\operatorname{st}(l,-1)+l=l=\frac{d}{3}=\left\lfloor\frac{d}{3}\right\rfloor=\left\lfloor\frac{d+1}{3}\right\rfloor .
$$

$$
\operatorname{st}(d, d-1)=\operatorname{st}(l+1,0)+l=l=\frac{d-1}{3}=\left\lfloor\frac{d-1}{3}\right\rfloor=\left\lfloor\frac{d+1}{3}\right\rfloor .
$$

If $d=3 l+2$, then

$$
\operatorname{st}(d, d-1)=\operatorname{st}(l+2,1)=l+1=\frac{d-2}{3}+1=\frac{d+1}{3}=\left\lfloor\frac{d+1}{3}\right\rfloor .
$$

Therefore we obtain

$$
\operatorname{st}(d, d-1)=\left\lfloor\frac{d+1}{3}\right\rfloor .
$$

Note that the recursive relations (4.7) can be rephrased as

$$
\operatorname{st}(d, j)= \begin{cases}\operatorname{st}(d+2, j+3)-1 & \text { if } j \leq d-2, \\ \operatorname{st}(d+2, j+3) & \text { if } j \geq d-1 .\end{cases}
$$

Hence if $j \geq d$, we have

$$
\operatorname{st}(d, j)=\operatorname{st}(d-2, j-3)=\operatorname{st}(d-4, j-6)=\cdots=\operatorname{st}(d-2 l, j-3 l)=\cdots
$$


Thus when we reach the point where $d-2 l-1=j-3 l . l=j-d+1$ and we have

$$
\operatorname{st}(d, j)=\operatorname{st}(d-2 l, j-3 l)=\left\lfloor\frac{d-2 l+1}{3}\right\rfloor=\left\lfloor\frac{3 d-2 j-1}{3}\right\rfloor .
$$

If $j \leq d-2$, then

$$
\operatorname{st}(d, j)=\operatorname{st}(d+2, j+3)-1=\operatorname{st}(d+4, j+6)-2=\cdots=\operatorname{st}(d+2 l, j+3 l)-l=\cdots
$$

. Thus when $d+2 l-1=j+3 l, l=d-j-1$ and we have

$$
\begin{aligned}
\operatorname{st} & (d, j)=\operatorname{st}(d+2 l, j+3 l)-l=\left\lfloor\frac{d+2 l+1}{3}\right\rfloor-l \\
& =\left\lfloor\frac{d+2(d-j-1)+1}{3}\right\rfloor-(d-j-1) \\
& =\left\lfloor\frac{3 d-2 j-1}{3}\right\rfloor-(d-j-1) .
\end{aligned}
$$

Note that the case $j=d-1$ is included in the above formula.

Therefore, the multiplicities of standard representations are given by the following theorem.

Theorem 5.7. For all $d \geq 3$ and $j \geq 0$, we have

$$
\operatorname{st}(d, j)= \begin{cases}\left\lfloor\frac{3 d-2 j-1}{3}\right\rfloor & \text { if } j \geq d, \\ \left\lfloor\frac{3 d-2 j-1}{3}\right\rfloor-(d-j-1) & \text { if } j \leq d-1 .\end{cases}
$$

Example 5.8. By (5.2) and Theorem 5.7, we have

$$
\begin{aligned}
& \operatorname{st}(9,8)=\left\lfloor\frac{9+1}{3}\right\rfloor=3, \quad \operatorname{st}(9,12)=\left\lfloor\frac{27-24-1}{3}\right\rfloor=0, \\
& \operatorname{st}(9,6)=\left\lfloor\frac{27-12-1}{3}\right\rfloor-(9-6-1)=4-2=2, \\
& \operatorname{st}(10,9)=\left\lfloor\frac{10+1}{3}\right\rfloor=3, \quad \operatorname{st}(10,12)=\left\lfloor\frac{30-24-1}{3}\right\rfloor=1, \\
& \operatorname{st}(10,6)=\left\lfloor\frac{30-12-1}{3}\right\rfloor-(10-6-1)=5-3=2 .
\end{aligned}
$$

Note that all the computations coincide with each other both in recursive form and in closed form.

\section{REFERENCES}

[1] W. Fulton, Young Tableaux, London Mathematical Society Student Texts 35, Cambridge University Press, 1997.

[2] W. Fulton and J. Harris, Representation Theory, A First Course, Reading in Mathematics 129, Springer-Verlag, New York, 2004.

[3] B. Harbourne, H. Schenck, and A. Seceleanu, Inverse systems, Gelfand-Tsetlin patterns and the weak Lefschetz property, J. London Math. Soc. 84 (2011), 712-730.

[4] T. Harima, T. Maeno, H. Morita, Y. Numata, A. Wachi, and J. Watanabe, The Lefschetz properties, Lecture Notes in Mathematics 2080, Springer-Verlag, Heidelberg, 2013.

[5] T. Harima, J. Migliore, U. Nagel, and J. Watanabe, The weak and strong Lefschetz properties for Anrinian K-algebras, J. Alg. 262 (2003), 99-126.

[6] J. Herzog and D. Popescu, Strong Lefschetz property and simple extensions, Available on the arXiv at http://front.math.ucdavis.edu/0506.5537.

[7] J. E. Humphreys, Introduction to Lie Algebras and Representation Theory, Graduate Texts in Mathematics 29, 2nd ed., Springer-Verlag, New York, 1978. 
[8] Y. R. Kim, and Y.-S. Shin, Representation theory of symmetric groups and the strong Lefschetz property: general case, in progress.

[9] A. Iarrobino, P. M. Marques, and C. McDaniel, Jordan type and the Associated graded algebra of an Artinian Gorenstein algebra, arXiv:1802.07383 (2018).

[10] T. Maeno, Lefschetz property, Schur-Weyl duality and a q-deformation of Specht polynomials, Comm. Algebra 35 (2007), 1307-1321.

[11] J. Migliore, R. Miró-Roig, and U. Nagel, Monomial ideals, almost complete intersections, and the weak Lefschetz property, Trans. Amer. Math. Soc. 363 (2011), 229-257.

[12] J. Migliore and U. Nagel, Survey Article: A tour of the weak and strong Lefschetz properties, J. Commut. Algebra 5 (2013), 329-358.

[13] L. Reid, L. Roberts, and M. Roitman, On complete intersections and their Hilbert functions, Canad. Math. Bull. 34 (1991), 525-535.

[14] B. E. Sagan, The Symmetric Group: Representations, Combinatorial Algorithms, and Symmetric Functions, Graduate Texts in Mathematics 203, 2nd ed., Springer-Verlag, New York, 2001.

[15] H. Schenck and A. Seceleanu, The weak Lefschetz property and powers of linear forms in $\mathbb{k}[x, y, z]$, Proc. Amer. Math. Soc. 138 (2010), 2335-2339.

[16] R. Stanley, Weyl groups, the hard Lefschetz theorem, and the Sperner property, SIAM J. Algebraic Discrete Methods 1 (1980), 168-184.

[17] J. Watanabe, The Dilworth number of Artinian rings and finite posets with rank function, Commutaive Algebra and Combinatorics, Advanced Studies in Pure Math. 11, Kinokuniya Co. North Holland, Armsterdam (1987), 303-312.

[18] H. Weyl, The classical groups: Their Invariants and Representations, Princeton Landmarks in Mathematics, Princeton University Press, Princeton, 1997.

Korea Research Institute of Arts and Mathematics, Asan-si, Chungcheongnam-do, 31551, Korea

E-mail address: soccerkang@hotmail.com

Major in Mathematics Education, Graduate School of Education, Hankuk University of Foreign Studies, Seoul, 02450, KOREA

E-mail address: rocky777@hufs.ac.kr

Department of Mathematics, Sungshin Women's University, Seoul, 02844, Korea and School of Mathematics, Korea Institute for Advanced Study, Seoul, 02455, Korea

E-mail address: ysshin@sungshin.ac.kr 